\title{
Targeted regulation of self-peptide presentation prevents type I diabetes in mice without disrupting general immunocompetence
}

\author{
Woelsung Yi,1 Nilufer P. Seth,2,3 Tom Martillotti, ${ }^{1}$ Kai W. Wucherpfennig, ${ }^{2,4}$ \\ Derek B. Sant'Angelo, ${ }^{1,5,6}$ and Lisa K. Denzin'1,5,6
}

\begin{abstract}
1 Immunology Program, Sloan-Kettering Institute, Memorial Sloan-Kettering Cancer Center, New York, New York. 2Department of Cancer Immunology and AIDS, Dana-Farber Cancer Institute, Boston, Massachusetts. ${ }^{3}$ Department of Pathology and ${ }^{4}$ Department of Neurology and Program in Immunology, Harvard Medical School, Boston, Massachusetts.

${ }^{5}$ Weill Graduate School of Medical Sciences of Cornell University, New York, New York.

${ }^{6}$ Louis V. Gerstner Jr. Graduate School of Biomedical Sciences, New York, New York.
\end{abstract}

\begin{abstract}
Peptide loading of MHC class II (MHCII) molecules is directly catalyzed by the MHCII-like molecule HLA-DM (DM). Another MHCII-like molecule, HLA-DO (DO), associates with DM, thereby modulating DM function. The biological role of DO-mediated regulation of DM activity in vivo remains unknown; however, it has been postulated that DO expression dampens presentation of self antigens, thereby preventing inappropriate $T$ cell activation that ultimately leads to autoimmunity. To test the idea that DO modulation of the MHCII selfpeptide repertoire mediates self tolerance, we generated NOD mice that constitutively overexpressed DO in DCs (referred to herein as NOD.DO mice). NOD mice are a mouse model for type 1 diabetes, an autoimmune disease mediated by the destruction of insulin-secreting pancreatic $\beta$ cells. Our studies showed that diabetes development was completely blocked in NOD.DO mice. Similar to NOD mice, NOD.DO animals selected a diabetogenic $T$ cell repertoire, and the numbers and function of Tregs were normal. Indeed, immune system function in NOD.DO mice was equivalent to that in NOD mice. NOD.DO DCs, however, presented an altered MHCII-bound self-peptide repertoire, thereby preventing the activation of diabetogenic $T$ cells and subsequent diabetes development. These studies show that DO expression can shape the overall MHCII self-peptide repertoire to promote $\mathrm{T}$ cell tolerance.
\end{abstract}

\section{Introduction}

Type 1 diabetes (T1D) is a chronic autoimmune disease mediated by the destruction of insulin-producing pancreatic $\beta$ cells by self-reactive $T$ cells. The self-reactive $T$ cells eventually mediate the destruction of enough pancreatic $\beta$ cells, ultimately leading to severe insulin deficiency. In NOD mice, the mouse model of T1D, defects in both central and peripheral $\mathrm{T}$ cell tolerance have been implicated in disease induction (1). The presentation of peptides derived from islet proteins bound to MHC class II (MHCII) molecules on the surface of DCs is essential for the maintenance of central and peripheral tolerance. Recognition of such complexes by self-reactive CD4 T cells normally leads to the deletion or functional inactivation of the self-referential $\mathrm{T}$ cell populations. Breakdown in tolerance mechanisms leads to autoimmunity.

The presentation of MHCII peptide complexes by DCs is important not only for central and peripheral $\mathrm{T}$ cell tolerance but also for the initial activation of naive CD4 T cells (2). Indeed, the activation of self-reactive $T$ cell responses that ultimately lead to $\beta$ cell destruction and T1D requires presentation of islet-derived antigens (Ags) by DCs $(3,4)$. Additionally, DC Ag presentation is thought to drive disease amplification that maintains the autoimmune response and results in $\beta$ cell destruction (5).

Conflict of interest: The authors have declared that no conflict of interest exists. Citation for this article: J Clin Invest. 2010;120(4):1324-1336. doi:10.1172/JCI40220.
Although genetic susceptibility to T1D is controlled by multiple loci in both humans and NOD mice, the major susceptibility locus is the MHC region, which accounts for approximately $50 \%$ of the total genetic contribution to T1D (6). NOD mice express an unusual I-A molecule (I-Ag7) that contains a nonaspartic acid substitution at position 57 of the $\beta$ chain. This polymorphism substantially alters the repertoire of presented peptides as compared with related alleles $(7,8)$. I-Ag7 expression is crucial for T1D development, in part because the altered I-A $\mathrm{A}^{7}$-bound peptide repertoire in NOD mice has been shown to mediate the selection of self-reactive T cells in the thymus (9). Significantly, this substitution is also seen in the human DQ $\beta$ chain, the human MHCII allele linked to T1D (10).

The molecular pathways by which MHCII molecules acquire peptide cargo have been examined in detail (reviewed in ref. 11). Briefly, newly formed MHCII $\alpha \beta$ heterodimers associate with the invariant chain (Ii) during their assembly in the ER. Ii occupies the peptide-binding groove of MHCII, preventing unfolded proteins in the ER from binding to MHCII molecules. Ii also functions to target MHCII-Ii complexes to late endosomal compartments in which Ii is degraded by resident proteases, leaving only small fragments of Ii, class II-associated Ii peptides (CLIP), in the MHCII peptide groove. Exchange of CLIP for peptides derived from self proteins and foreign Ags is catalyzed by the action of the MHCIIlike molecule H2-M (HLA-DM in humans [DM]). H2-M also functions as a peptide editor and an MHCII-specific chaperone that 
stabilizes peptide-receptive MHCII. Following peptide binding, the resultant MHCII peptide complexes are transported to the cell surface for presentation to CD4 T cells.

Peptide loading of MHCII molecules is modulated in DCs, B cells, and medullary thymic epithelial cells by the association of another class II-like molecule, HLA-DO (DO; H2-O in mice) with $\mathrm{DM} / \mathrm{H} 2-\mathrm{M}(12-17)$. DM/DO (H2-M/H2-O) association is initiated in the ER and maintained during and after transport to endosomal compartments in which the DM/DO complex resides (18). The tight association of DM with DO modulates the peptide-loading function of DM, resulting in an altered MHCII-bound peptide repertoire (19). Importantly, DO/H2-O is downregulated upon APC activation, freeing DM/H2-M from DO/H2-O inhibition, presumably resulting in an optimally active MHCII peptide-loading pathway upon pathogen encounter in vivo $(15,17,20-22)$.

DO expression in nonactivated APCs has been suggested to generate a broad, tolerogenic MHCII-bound peptide pool by dampening $\mathrm{DM} / \mathrm{H} 2-\mathrm{M}$ activity $(12,23)$. Thus, $\mathrm{DO} / \mathrm{H} 2-\mathrm{O}$ expression potentially has an important role in preventing autoimmune diseases such as T1D by promoting central and peripheral T cell tolerance. Data supporting this idea, however, are lacking. Ag presentation by DCs is known to be essential for T1D disease initiation and propagation (3, 4). Therefore, to directly assess the role of $\mathrm{DO} / \mathrm{H} 2-\mathrm{O}$ in altering the MHCII peptide repertoire and the impact this would have on T1D, diabetes-prone NOD mice that express human DO in DCs were generated. Our studies showed that diabetes development was completely blocked by DO expression in DCs. Protection was most likely not due to a change in central tolerance, nor was it likely mediated by an alteration in regulatory $\mathrm{T}$ cell function. T1D protection was due to the inefficient presentation of self Ag by DO-overexpressing DCs to autoreactive T cells. These studies provide direct evidence that $\mathrm{DO} / \mathrm{H} 2-\mathrm{O}$ maintains peripheral tolerance and suggest a novel therapeutic approach for the prevention and treatment of T1D.

\section{Results}

NOD mice overexpressing DO in DCs are protected from $T 1 D$. The initial activation and subsequent maintenance of islet-specific $T$ cell responses that ultimately leads to autoimmune diabetes is driven by DCs displaying MHCII molecules complexed with peptides derived from islet-derived self proteins $(3,4)$. Previous studies from our lab showed that MHCII self peptide presentation on the surface of C57BL/6, B10.BR, and C57BL/ $6 \times$ B10.BR F FCs was $_{1}$ modulated by the expression of DO (24). DO expression in the CD11c-DO Tg mice was achieved by placing the human DOA and $\mathrm{DOB}$ genes under the control of the DC-specific CD11c promoter, which resulted in impaired H2-M function and altered MHCII Ag processing and presentation (24). Human DO and mouse H2-O are highly conserved, and thus the human proteins were used to generate these transgenic mice to allow us to take advantage of antibodies specific for the human DO heterodimer. To determine whether DO-mediated modulation of the MHCII peptide repertoire could impact T1D, the CD11c-DO Tg mice were crossed onto the NOD background for more than 15 generations (NOD.DO). Microsatellite analysis confirmed that Idd-recessive loci were NOD derived (data not shown). For all studies in which NOD.DO mice were compared with NOD mice, non-Tg littermate control mice were used and will be referred to in the text hereafter as NOD mice.

As expected, nearly all CD $11 \mathrm{c}^{+} \mathrm{DCs}$ in NOD.DO mice expressed the transgene-encoded DO (Figure 1A). In addition, a fraction of the marginal zone $(\mathrm{MZ})$ and follicular $(\mathrm{FO}) \mathrm{B}$ cells were also found to express the protein (Figure 1B). Activated B cells have been shown to express CD11c, potentially explaining the expression of the DO transgene in a subset of total B cells (25). DO expression was not observed in any other cell types. Overall DC development was normal in NOD.DO mice (Supplemental Figure 1; supplemental material available online with this article; doi:10.1172/JCI40220DS1), and NOD and NOD.DO DCs had similar levels of CD80, CD86, CD40, and ICAM-1, indicating that DC activation was not altered (data not shown). Additionally, no major differences in the percentages and absolute numbers of CD4 and CD8 T cells or FO and MZ $\mathrm{B}$ cells were observed in the thymus, spleen, and pancreatic lymph nodes (PLN) of NOD.DO mice (Supplemental Figure 2 and data not shown). Finally, the expression of CD44, CD69, and CD62L on $\mathrm{T}$ cells was unaltered in NOD.DO mice, showing that the overall $\mathrm{T}$ cell activation was normal (data not shown).

To determine whether DO expression in DCs would alter diabetes development, age-matched female NOD.DO and littermate control non-Tg NOD mice were tested for diabetes onset by monitoring urine glucose levels for 50 weeks. As shown in Figure 1C, diabetes onset in NOD mice first occurred at 17 weeks of age, and $94 \%$ were diabetic by 50 weeks. In contrast, NOD.DO mice did not develop diabetes; all mice remained diabetes free throughout the 50-week period of analysis. To rule out aberrant insertion of the transgenes into a loci that alters immune cell function as the mechanism of protection from diabetes, an additional line of NOD.DO mice was generated by coinjection of the DO transgenes directly into NOD blastocysts. This additional line of NOD. DO mice (NOD.DO2) also remained diabetes free (Supplemental Figure 3). These data suggested that DO expression in NOD DCs provided a powerful means to downmodulate or alter the presentation of islet-derived peptides bound to MHCII molecules, thereby preventing T1D.

NOD.DO mice are protected from insulitis. The initial priming of autoreactive T cells that cause T1D has been shown to occur in the PLN (26). This event is followed by periislet infiltration and insulitis (1). To determine whether DO expression prevents islet infiltration, a histological analysis of pancreata from female NOD. $\mathrm{DO}$ and $\mathrm{Tg}$-negative littermate control NOD mice for evidence of periinsulitis and insulitis at 5, 10, 15, 25, and 35 weeks of age was performed. Representative sections show that insulitis and islet destruction were observed in control NOD mice starting by 10 weeks of age (Figure 2A). Quantification of histological sections showed that $70 \%, 52 \%$, and $47 \%$ of the islets exhibited severe insulitis at 15, 25, and 35 weeks, respectively (Figure 2B). The lower percentages of insulitis in aged NOD mice are due to the early death of severely diabetic mice. In contrast, islets were well preserved in NOD.DO mice (Figure 2A). Only 5\%-7\% of the islets showed aggressive infiltration by 15 weeks (Figure 2B). Remarkably, even 35 -week-old mice had very little insulitis (Figure 2B). Although NOD.DO mice exhibited almost no insulitis, periinsulitis was observed in a similar percentage of control NOD and NOD.DO mice throughout the 35-week analysis (Figure 2B).

The near complete destruction of islets seen in NOD mice was essentially completely prevented in NOD.DO mice (Figure 2C). By 25 weeks, for example, many of the NOD mice had lost nearly all of their islets, while the number of islets in NOD.DO mice was maintained. Collectively, these data show that NOD.DO mice have periinsulitis similar to NOD mice, but that the initial nondestructive periinsulitis does not progress to the destructive insulitis that ultimately leads to overt diabetes. 
A

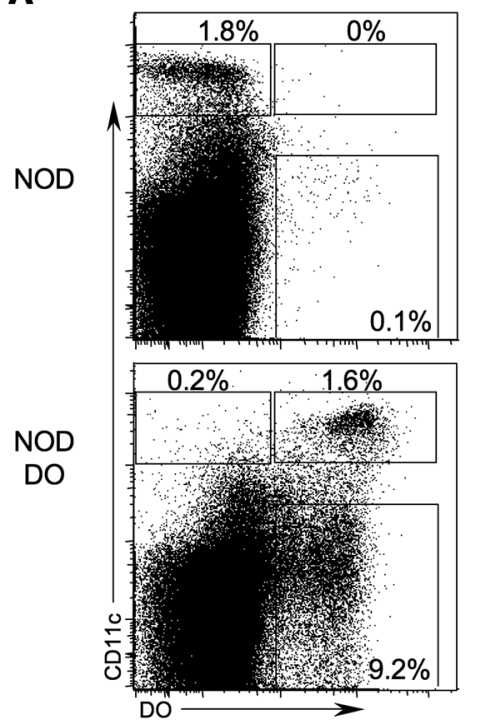

B

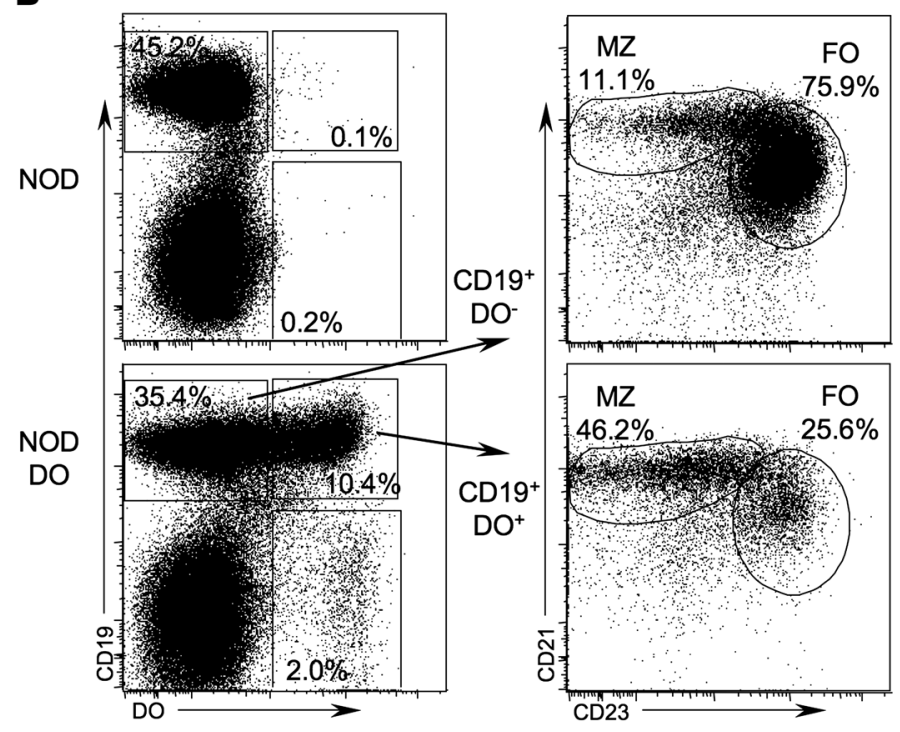

C

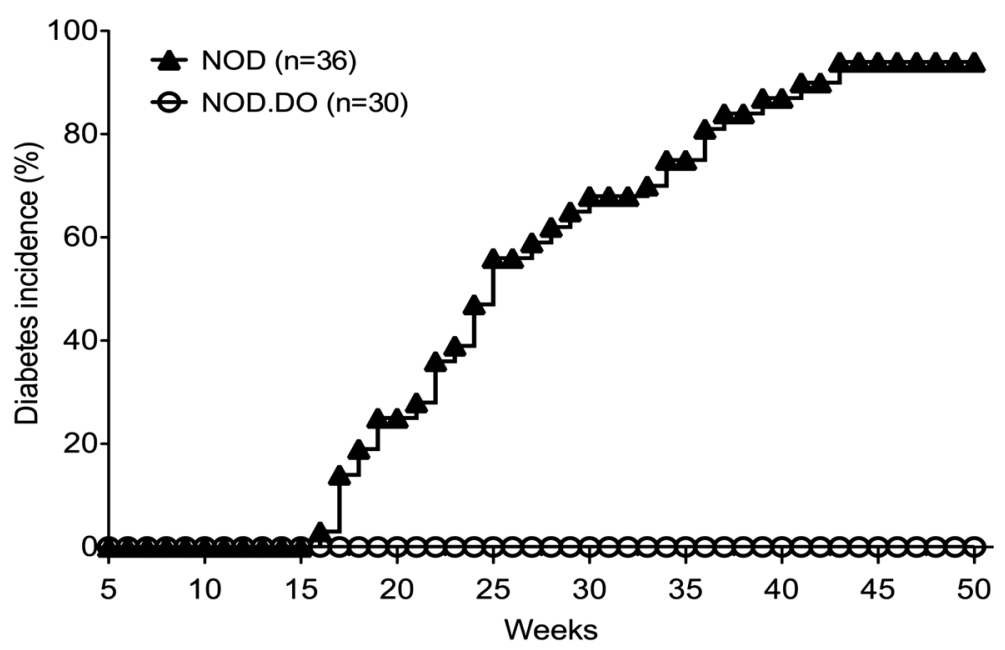

Figure 1

NOD.DO mice do not develop diabetes. (A) Splenocytes from NOD and NOD.DO mice were stained with a mAb specific for CD11c and then stained intracellularly with a mAb specific for DO prior to analysis by flow cytometry. The mAb used for these analyses does not recognize endogenous mouse $\mathrm{H} 2-\mathrm{O}$. The numbers indicate the percentage of cells falling within each gated region. Data are representative of more than 20 independent experiments. (B) Splenic B cells from NOD and NOD.DO mice were stained with mAbs to CD19, CD21, and CD23 to identify FO $\left(\mathrm{CD} 19^{+} \mathrm{CD} 21^{10} \mathrm{CD} 23^{+}\right)$and $\mathrm{MZ}\left(\mathrm{CD} 19^{+} \mathrm{CD} 21^{\mathrm{hi}} \mathrm{CD} 23^{\circ}\right) \mathrm{B}$ cells and then stained intracellularly with a mAb to DO. Left plots show total splenocytes. Right plots are gated on the $\mathrm{DO}^{+}\left(\mathrm{CD} 19^{+} \mathrm{DO}^{+}\right)$and $\mathrm{DO}^{-} \mathrm{B}$ cells $\left(\mathrm{CD} 19^{+} \mathrm{DO}^{-}\right)$and further fractionated into $\mathrm{MZ}$ and $\mathrm{FO}$ B cell populations. The numbers indicate the percentage of cells falling within each gated region. Data are representative of more than 20 independent experiments. (C) Diabetes frequency was determined by measurement of urine glucose levels in female NOD.DO and non-Tg littermate control NOD mice. Mice with 2 consecutive measurements exceeding $250 \mathrm{mg} / \mathrm{dl}$ were considered diabetic, with the first positive reading scored as diabetic.

NOD.DO mice have a diabetogenic T cell repertoire and functional Tregs. CD $11 \mathrm{c}^{+}$DCs located in the medulla of the thymus contribute to the negative selection of self-reactive $T$ cells either by cross presentation of tissue-specific Ags derived from medullary thymic epithelial cells or by direct presentation of tissue-specific Ags expressed in DCs $(27,28)$. Hence, it is possible that DO expression in thymic DCs alters $T$ cell-negative selection such that pathogenic $T$ cells are eliminated during $\mathrm{T}$ cell development in NOD.DO mice, thereby resulting in protection from diabetes.

Thus, to determine whether T cells from NOD.DO mice had diabetogenic potential, purified splenic T cells from 22-week-old
NOD.DO mice were transferred into NOD.scid recipients. Old NOD.DO mice were used to increase the likelihood that there had been activation of pathogenic T cells. As a control, purified $\mathrm{T}$ cells from age-matched NOD mice were also transferred. All of the recipient mice that received $\mathrm{T}$ cells from NOD mice developed diabetes by 7 weeks after transfer (Figure 3A). In contrast, none of the mice that received T cells from NOD.DO mice had developed diabetes at the same time point. However, by 10 weeks, $60 \%$ of the recipients that received T cells from NOD.DO mice developed diabetes. Diabetes development, albeit delayed, clearly demonstrates the presence of pathogenic T cells in NOD.DO 
A
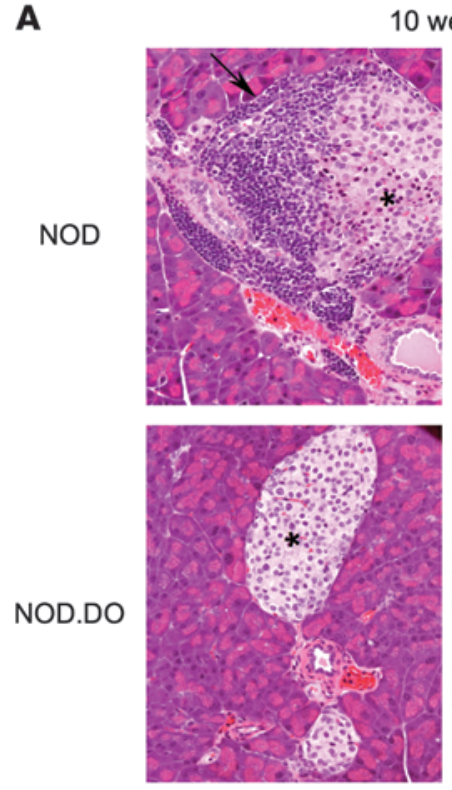

\section{weeks}
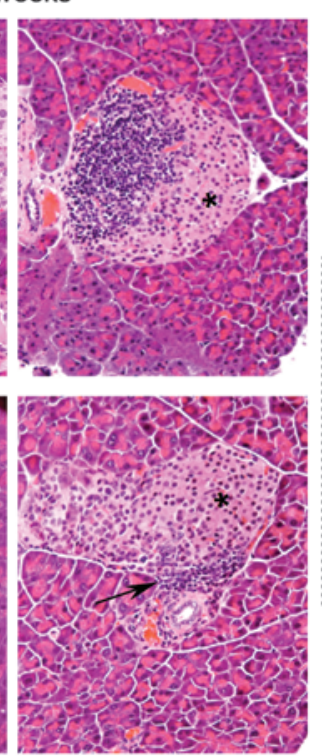

15 weeks
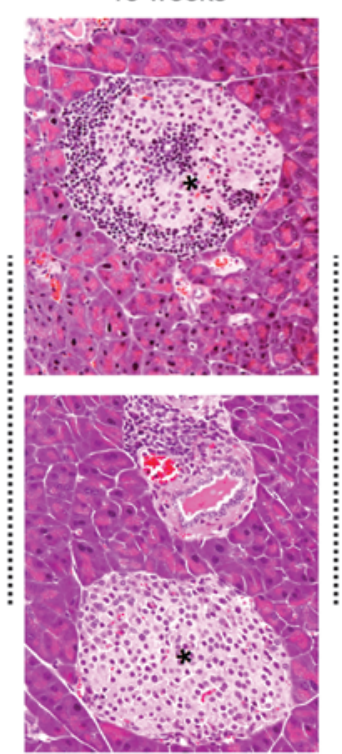

$\square$ periinsulitis

insulitis

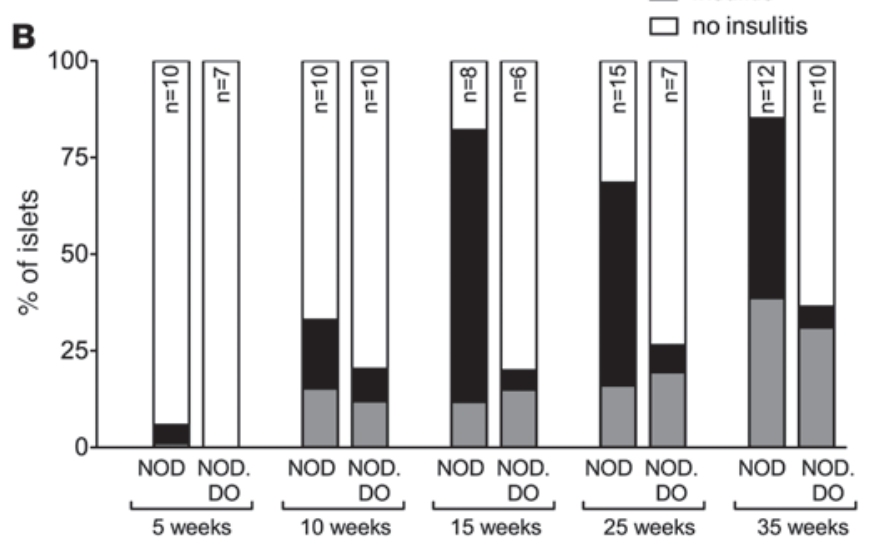

C

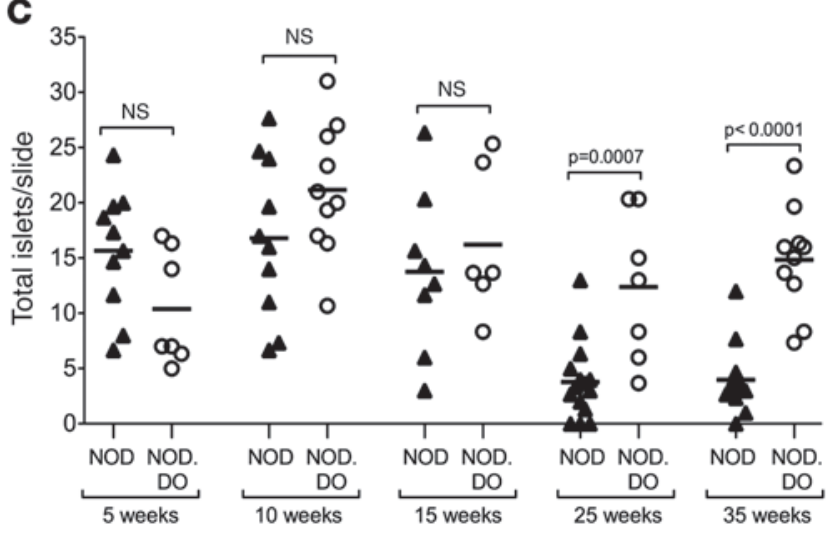

35 weeks

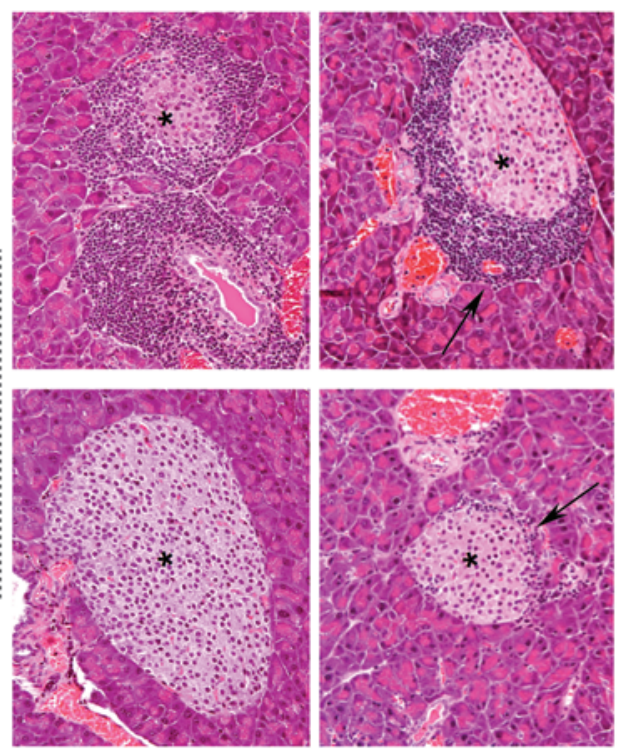

Figure 2

NOD.DO mice have low levels of insulitis. (A) Representative pancreatic sections from 10-, 15-, and 35-week-old female NOD and NOD.DO mice. Islets are marked with asterisks. Periinsulitis is indicated by arrows. Original magnification, $\times 20$. (B) The percentage of mice exhibiting insulitis or periinsulitis at 5, 10, 15, 25, and 35 weeks of age. Number of mice analyzed for each time point is indicated, and 3 slides/mouse were analyzed. (C) Average number of islets in NOD and NOD.DO pancreata in 5-, 10-, 15-, 25-, and 35-week-old mice for the mice analyzed in B. Each symbol represents an individual mouse, and small horizontal bars indicate the mean. $P$ values were computed using 2-tailed, unpaired Student's $t$ test.

mice. The transfer of naive (prediabetic) T cells into NOD.scid recipients has been shown to dramatically delay diabetes onset (29). Thus, the delayed onset of diabetes in NOD.DO T cell recipients is most likely explained by lack of sufficient pathogenic $\mathrm{T}$ cell priming in NOD.DO mice.

A second, albeit less likely possibility is that there was enhanced Treg function in NOD.DO mice. DO expression in DCs did not alter overall Treg selection and development, as NOD and NOD. DO mice have similar percentages and numbers of thymic, splenic, and PLN Tregs (Supplemental Figure 4 and data not shown). The higher numbers of Tregs observed in the thymus and spleen of NOD.DO mice compared with NOD mice is most likely due to diabetes onset in the older mice and is unlikely due to DO expression (Supplemental Figure 4). To further evaluate Treg function, CD25-depleted T cells from 22-week-old NOD and NOD.DO mice were transferred into NOD.scid recipients. Depletion of CD25+ T cells resulted in faster kinetics of diabetes development in recipients that received $\mathrm{T}$ cells from either NOD or NOD.DO mice (Figure 3B), demonstrating that Tregs in NOD.DO mice are capable of suppressing islet-specific T cell responses. Importantly, the removal of Tregs from NOD.DO T cells did not revert diabetes development to the time frame observed when $\mathrm{CD} 25^{+} \mathrm{T}$ cells were depleted from NOD T cells. Thus, DO expression did not promote the development of a Treg population harboring enhanced function. Together, these data suggest that NOD.DO mice have normal Tregs and a pathogenic $\mathrm{T}$ cell repertoire; however, the pathogenic $T$ cells are not activated, as evident from the delayed disease onset. These data suggest that peripheral tolerance is the likely mechanism for protection from diabetes in NOD.DO mice, since the mice fail to develop diabetes despite the presence of pathogenic $\mathrm{T}$ cells. 

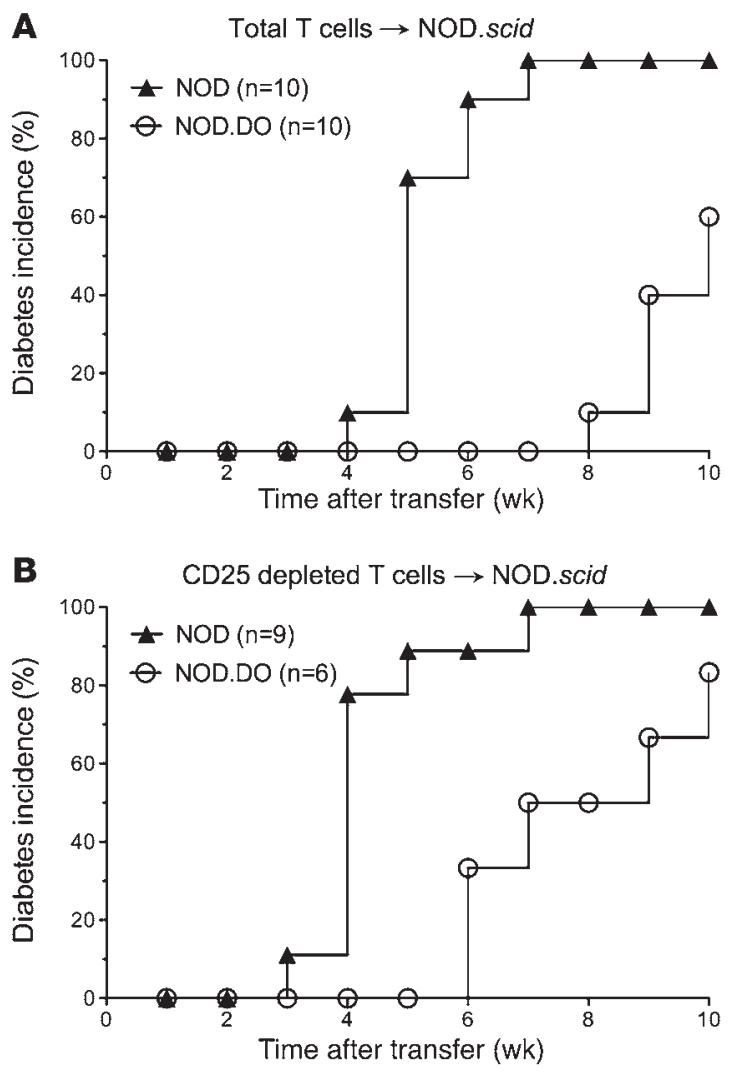

However, these studies are not quantitative and therefore cannot exclude the alternative possibility that delayed diabetes onset is due to reduced numbers of pathogenic CD4 T cells or pathogenic CD4 T cells with altered Ag specificities in NOD.DO mice.

DO expression in DCs does not prevent the selection, development, and function of islet-specific T cells. I-A ${ }^{7}$ MHC tetramers loaded with the peptide mimitope 2.5 (A $\mathrm{g}^{7} / \mathrm{BDC} 13$; AAVRPLWVRMEAA) specifically bind to the TCR expressed by BDC2.5 Tg T cells, which express a TCR specific for an unknown islet Ag (30). $\mathrm{A}^{\mathrm{g}}$ /BDC13 tetramers also stain a distinct population of peripheral BDC2.5-like CD4 T cells in non-Tg NOD mice (30). Accordingly, to determine whether islet-specific $\mathrm{T}$ cells are present in NOD.DO mice, PLN T cells from NOD.DO and wild-type NOD mice were stained with $\mathrm{Ag}^{\mathrm{g}} / \mathrm{BDC} 13$ tetramers. $\mathrm{A}^{\mathrm{g}} / \mathrm{CLIP}$ tetramers were used as a negative control, since this is an endogenous $\mathrm{Ag}$ that is expressed in the thymus, but $T$ cells recognizing this self peptide are efficiently negatively selected and thus, are not present (30). In PLNs pooled from mice, very few $\mathrm{A}^{\mathrm{g} 7} / \mathrm{CLIP}$-reactive CD4 T cells were found in NOD or NOD.DO mice, as expected (Figure 4A). However, similar numbers of tetramer-positive CD4 $T$ cells were detected in the PLNs from both NOD and NOD.DO mice. The presence of peripheral $\mathrm{A}^{\mathrm{g} 7} / \mathrm{BDC} 13$ tetramer-reactive CD4 T cells and the efficient selection of BDC2.5 TCR Tg T cells in NOD.DO mice show that islet-specific CD4 T cells could be positively selected in NOD.DO mice. These studies further support that overall $\mathrm{T}$ cell selection and function are not globally altered in NOD.DO mice.

NOD.DO mice were crossed with BDC2.5 TCR Tg mice (BDC2.5/NOD.DO) to determine whether thymocytes expressing this islet Ag-specific TCR could be selected (31, 32). As

\section{Figure 3}

NOD.DO mice have a diabetogenic $T$ cell repertoire and functional Tregs. (A) Purified splenic T cells $\left(5 \times 10^{6} /\right.$ mouse) from 22 -week-old female NOD or NOD.DO mice were transferred into female NOD.scid mice. Recipient mice were monitored for diabetes development by measurement of urine glucose levels. (B) As in A, except CD25depleted $T$ cells were transferred into recipients that were injected with $\alpha-C D 25$ at the time of transfer.

shown in Figure 4B, BDC2.5 T cell selection was unaltered by DO expression in DCs, as was evident by the accumulation of CD4 T cells in both the thymus and spleen of BDC2.5/NOD.DO and BDC2.5/NOD animals.

To show that the BDC2.5 TCR Tg T cells that developed in the NOD.DO mice were functional and not rendered nonresponsive by the DO expression in DCs, CFSE-labeled T cells from BDC2.5/NOD. $\mathrm{DO}$ and control BDC2.5/NOD mice were transferred into NOD recipients. Purified T cells were transferred to remove any DO-overexpressing DCs that might influence Ag presentation in the recipient mice. Three days after transfer, the proliferation of the transferred $\mathrm{T}$ cells was measured by progressive CFSE dilution. BDC2.5 T cells from NOD.DO and NOD mice proliferated equally well in the PLN, but did not proliferate in other LNs of the recipient mice (Figure 4, C and D). Therefore, BDC2.5 T cells that developed in NOD.DO mice proliferated in response to specific MHCII peptide and were functional. Collectively, these studies show that BDC2.5-like and BDC2.5 TCR Tg T cells are subjected to central tolerance in NOD.DO mice and that the TCR Tg T cells selected in these mice are functional. However, since the islet Ag recognized by this TCR is unknown, it is not clear that the $\mathrm{Ag}$ represents a self $\mathrm{Ag}$ that is essential for disease development. Indeed, below we present data that suggest the Ag is not important (Supplemental Figure 5). Nevertheless, these studies show that DO expression does not have an impact on the selection and function of T cells specific for at least one islet Ag.

DO dampens the presentation of islet-derived autoAgs. The transfer of pathogenic T cells from diabetic NOD mice into prediabetic NOD mice results in rapid diabetes induction (29). Thus, we reasoned that if DO prevents the presentation of relevant islet-derived autoAgs by DCs, then when challenged with pathogenic T cells, NOD. DO mice would either show delayed diabetes development or would be protected. To test this idea, purified splenic T cells from recently diabetic NOD mice ( $<2$ weeks after scoring positive for $>250 \mathrm{mg} / \mathrm{dl}$ glucose in the urine) were transferred into sublethally irradiated 7- to 8-week-old NOD or NOD.DO recipients (Figure 5A). As expected, control NOD recipients rapidly developed diabetes, beginning at 2 weeks after transfer, with all mice diabetic by 12 weeks (Figure 5B). In contrast, diabetes development in NOD.DO recipient mice was dramatically delayed (Figure 5B). NOD.DO recipients did not develop diabetes until 10 weeks after transfer at a point when $93 \%$ of control NOD recipients were already diabetic. Even at 15 weeks after transfer, nearly half of the NOD.DO recipients remained diabetes free. These data strongly suggest that in NOD. DO mice, DC-mediated presentation of islet-derived self-Ags to pathogenic $\mathrm{T}$ cells was reduced.

DO expression in NOD.DO mice is not restricted to DCs. A fraction of $\mathrm{MZ}$ and FO B cells also express DO (Figure 1B). To determine whether DO expression only in DCs was sufficient to protect NOD mice from diabetes, pathogenic T cells from recently diabetic NOD mice were transferred into RAG-1-deficient NOD (NOD.RAG1) and NOD.DO (NOD.RAG1.DO) mice (Figure 5C). 
A
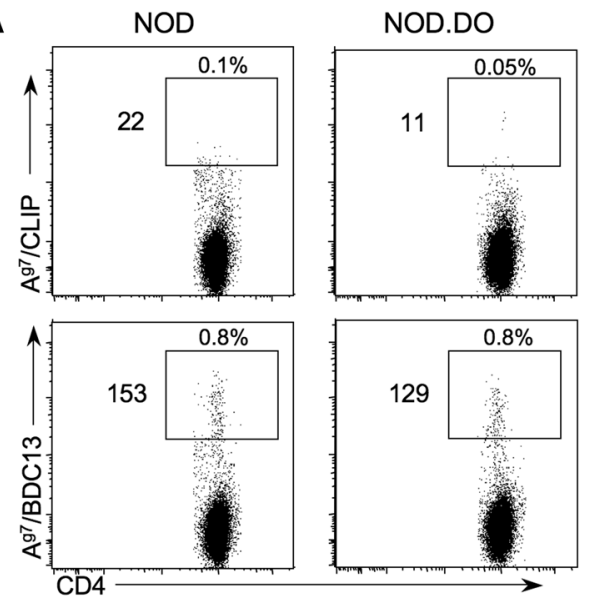

C

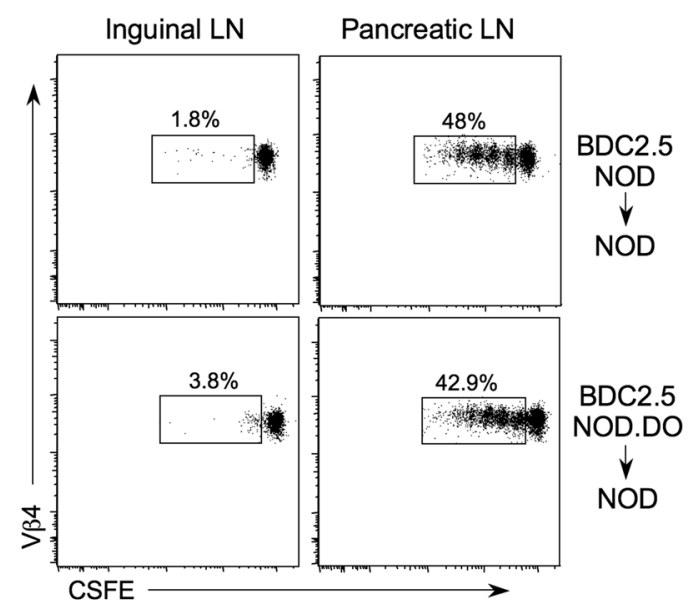

B

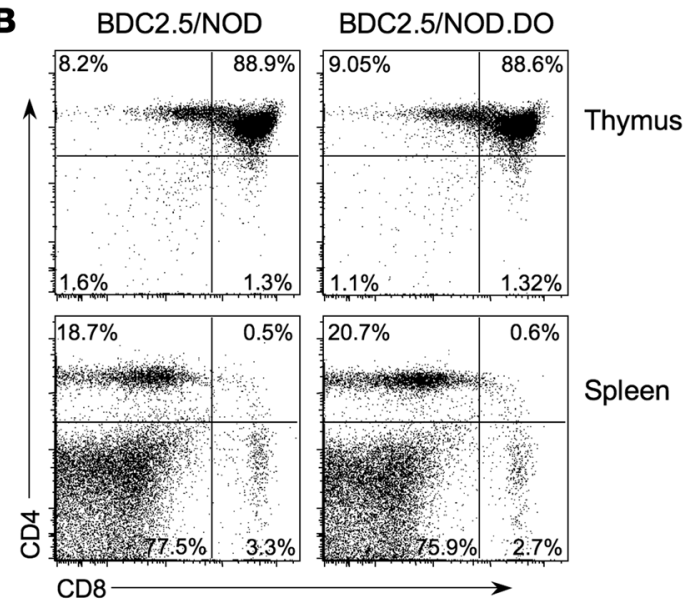

D

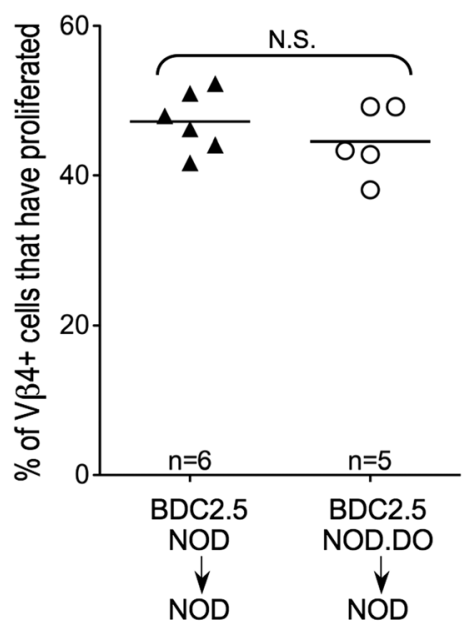

Figure 4

Selection and function of BDC2.5 T cells and BDC2.5-like T cells is unperturbed in NOD.DO mice. (A) PLN cells pooled from 5- to 7-week-old NOD and NOD.DO mice were stained with PE-labeled I-A97/CLIP or I-A97/BDC13 tetramers and APC-labeled anti-CD4 and tetramer-positive cells were enriched with anti-PE microbeads. Plots show tetramer versus CD4 after gating for $C D 3^{+} C D 4^{+} B 220^{-} C D 8^{-}$. Percentages on top of gates are percentages of $\mathrm{CD}^{+}{ }^{+}$tetramer ${ }^{+}$cells and numbers to the left are absolute numbers of $\mathrm{CD}^{+}{ }^{+}$tetramer ${ }^{+}$cells. These numbers were derived after analyzing $40 \%$ of the PLN lymphocytes pooled from 5 NOD or NOD.DO mice. Therefore, each mouse had approximately 70 BDC2.5-like T cells in its PLN. Data are representative of 3 independent experiments. (B) FACs analysis of thymocytes (top) and splenocytes (bottom) from BDC2.5/NOD and BDC2.5/NOD.DO mice. Numbers in quadrants indicate percentage of total cells falling within each gate. Data are representative of 4 to 5 total mice/genotype analyzed in 2 independent experiments. (C) BDC2.5 TCR Tg T cells from NOD.DO mice proliferate in response to $\mathrm{Ag}$ in vivo. CFSE-labeled BDC2.5 T cells from NOD or NOD.DO mice were transferred into NOD recipients, and 3 days later, CFSE dilution was monitored for the CD4+V $\beta 4^{+} \mathrm{T}$ cells in pancreatic and inguinal LNs of recipient mice. Numbers next to gates are the percentage of total $\mathrm{CFSE}^{+} \mathrm{CD} 4^{+} \mathrm{V} \beta 4^{+}$cells that underwent at least one round of proliferation. (D) Quantification of data in C for multiple mice. Each symbol represents an individual mouse, and small horizontal bars indicate the mean. One of 2 independent experiments is shown.

Since RAG-1-deficient mice lack B cells, this allowed us to assay islet Ag presentation only by DCs. As expected, control NOD. RAG1 recipients rapidly developed diabetes, beginning at 4 weeks after transfer, with $90 \%$ of the mice diabetic at 9 weeks (Figure 5D). In contrast, NOD.RAG1.DO recipient mice did not develop diabetes during the 9-week monitoring period (Figure 5D). These data further support that islet Ag presentation was reduced in DO-expressing mice. Furthermore, these studies show that the expression of DO only in DCs is sufficient to protect NOD mice from diabetes in a transfer system.

The experiments described above show that the overall I-Ag7 peptide repertoire is altered in NOD.DO mice, supporting that protection from diabetes is mediated by decreased presentation of islet-derived Ags by NOD.DO DCs. Therefore, to determine whether DO expression prevented the presentation of a specific islet $\mathrm{Ag}$ by DCs located in the PLN, we performed an in vivo Ag presentation assay. Purified BDC2.5 TCR Tg T cells from NOD mice were labeled with CFSE and transferred into NOD and NOD.DO recipient mice, and $T$ cell proliferation of the transferred T cells was measured 3 days later by monitoring CSFE dilution. BDC2.5 T cells proliferated equally in the draining PLN of NOD and NOD.DO recipients (Supplemental Figure 5). These data show that DO expression does not inhibit the activation and expansion of at least one pathogenic $T$ cell population. Since NOD.DO mice are protected from diabetes, DO expression may inhibit the presentation of a diabetes-initiating Ag (or Ags). Thus, 
A

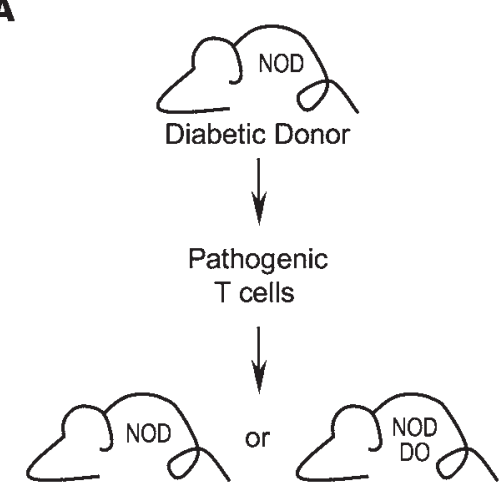

C

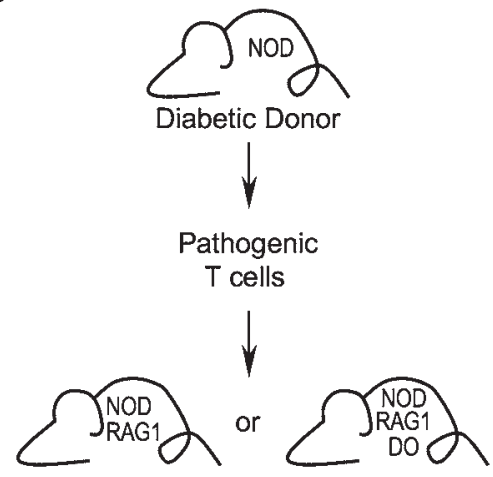

B

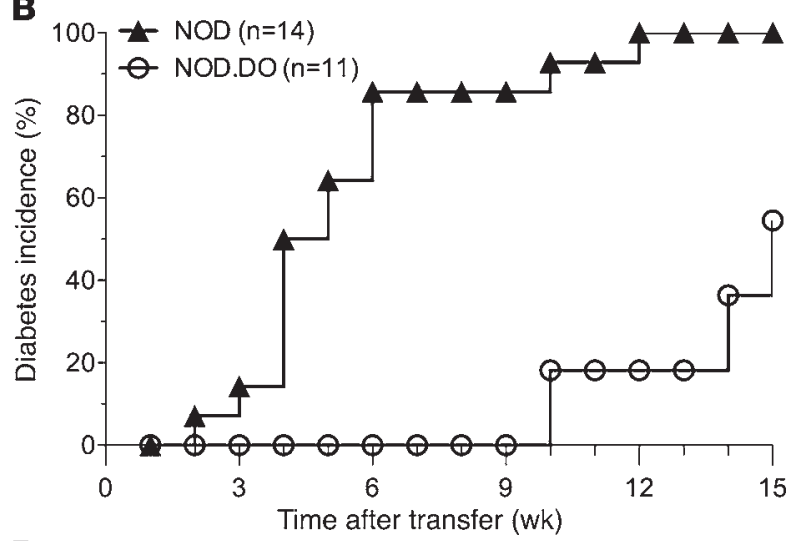

D

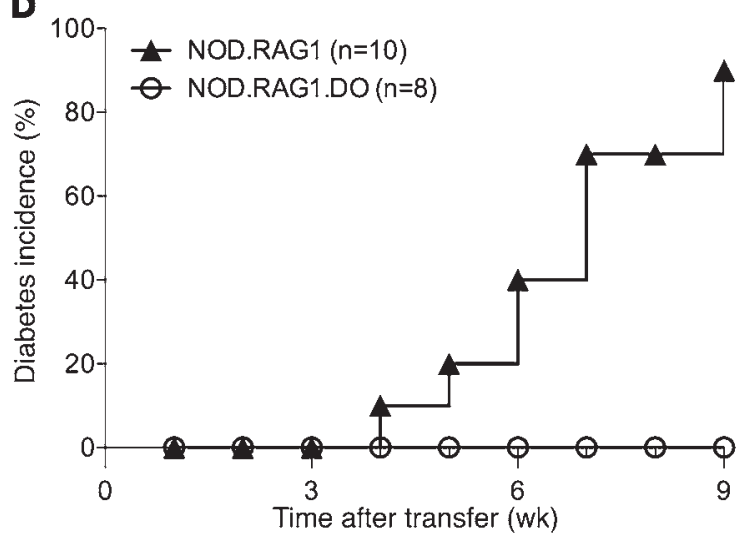

\section{Figure 5}

DO dampens the presentation of islet-derived autoAgs. (A and C) Schematic illustrating the experimental design. (B and D) Splenic T cells $\left(5 \times 10^{6}\right)$ purified from recently diabetic female NOD mice were transferred into sublethally irradiated NOD or NOD.DO recipients $(B)$ or nonirradiated NOD.RAG1 or NOD.RAG1.DO recipients (D). Recipient mice were monitored for diabetes development by measurement of urine glucose levels. Data were pooled from 2 (D) or 3 (B) independent experiments.

these data also support that the Ag recognized by the BDC2.5 TCR specificity is not likely to be a diabetes-initiating Ag.

Importantly, these data also show that DO expression does not induce a state of generalized immunosuppression, since pathogenic BDC2.5 T cells proliferated normally in NOD.DO mice. This is a key issue, as DO expression in DCs could inhibit overall Ag presentation, which in turn would create a state of generalized immunosuppression resulting in protection from diabetes. To further evaluate this possibility, control NOD and NOD.DO mice were immunized with the $\mathrm{T}$ cell-dependent Ag, NP-CGG, in alum and sera from the mice was monitored for the presence of NP-specific IgM and IgG. Results showed that there was no significant difference in the generation of NP-specific Ab responses in NOD and NOD.DO mice (Figure 6). These data are consistent with other studies from our lab showing normal antibody responses to OVA, conalbumin, and hen egg lysosome (HEL) in C57BL/6 mice overexpressing DO in DCs (L.K. Denzin and H.M.T. O'Rouke, unpublished observations).

NOD.DO DCs display an altered MHCII peptide repertoire. The binding of some mAbs to MHCII molecules has been shown to be peptide dependent (33-39). Therefore, we evaluated I- $\mathrm{A}^{7}$ expression levels on splenic DCs in NOD and NOD.DO mice using a panel of mAbs that recognize I-A $\mathrm{A}^{\mathrm{g} 7}$ : 10.2-16 (40), OX-6 (41), and AMS-32.1 (42).
The recognition of $\mathrm{I}-\mathrm{A}^{\mathrm{g} 7}$ on the cell surface of splenic DCs from NOD.DO mice by all $3 \mathrm{mAbs}$ was dramatically reduced relative to the levels on NOD DCs (Figure 7A). The reactivity of mAbs 10-2.16, OX-6, and AMS-32.1 to DCs from NOD.DO mice was reduced to $78 \%, 42 \%$, and $22 \%$, respectively, relative to the levels obtained for NOD DCs (Figure 7B). The reduced mAb reactivity to NOD.DO DCs was not due to sequestration of I-A $\mathrm{g}^{7}$ molecules in intracellular compartments, since the reactivity of mAbs 10-2.16 and OX-6 after fixation and permeabilization was also reduced for NOD.DO DCs (Figure 7, A and B). The epitope recognized by mAb AMS-32.1 was destroyed by fixation, so this $\mathrm{mAb}$ could not be used to measure total I-Ag7 reactivity. MHCII expressed by B cells from NOD and NOD.DO mice was, however, recognized at a similar level by all $3 \mathrm{mAbs}$ (Figure 7, C and D). Interestingly, $\mathrm{DO}^{+}$and $\mathrm{DO}^{-} \mathrm{B}$ cells from NOD.DO mice were also recognized to a similar degree by all $3 \mathrm{mAbs}$ (Figure 7, C and D), presumably due to approximately 2 -fold lower DO expression in B cells than DCs in NOD.DO mice (Figure 7E). Immunoblot analysis of detergent lysates from purified NOD and NOD.DO splenic DCs using a polyclonal rabbit serum to the cytoplasmic tail of I-A $\beta$ showed that overall I-As ${ }^{7}$ levels are similar in NOD and NOD.DO DCs (Figure 8A). Thus, the reduced reactivity of mAbs 10-2.16, OX-6, and AMS-32.1 to NOD. DO DCs supports that DO expression alters the I- $\mathrm{A}^{\mathrm{g} 7}$-bound peptide repertoire presented on the surface of NOD.DO DCs. 
A
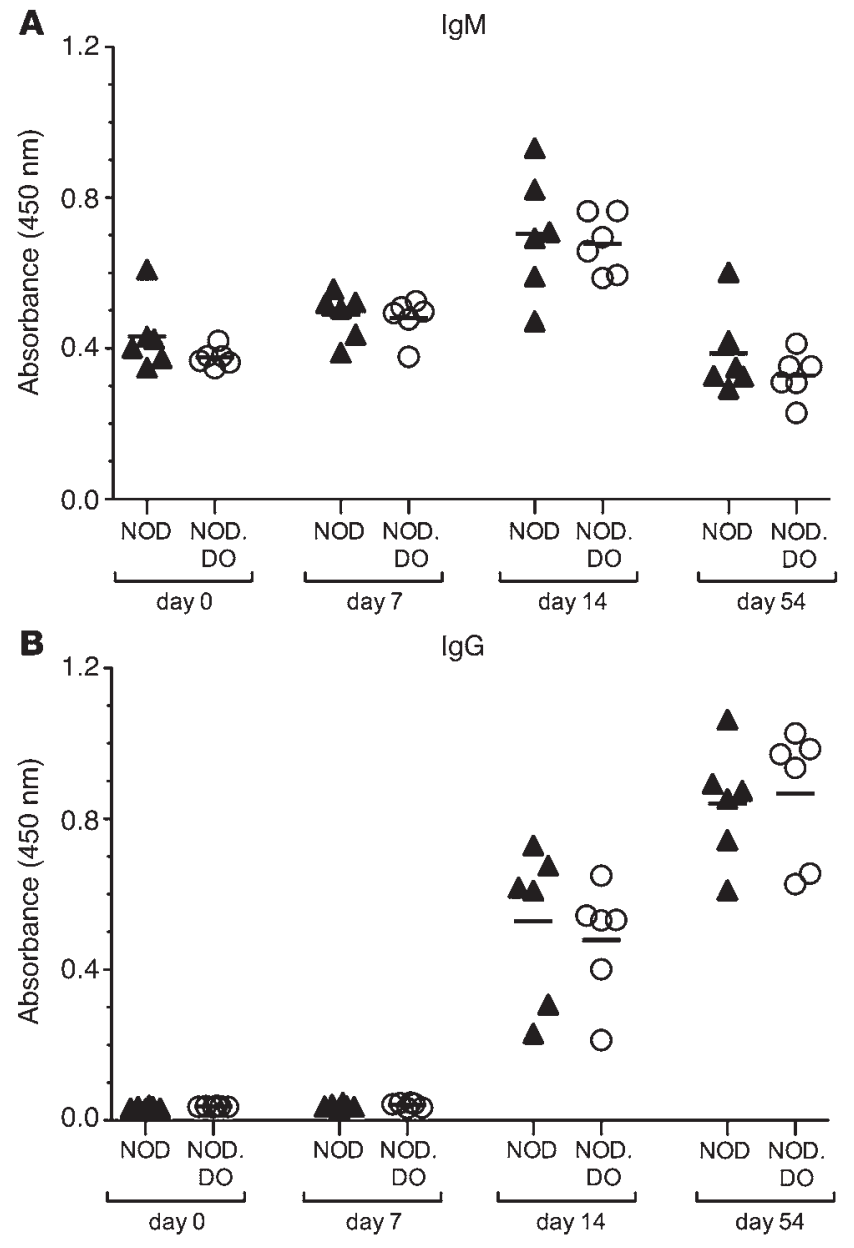

A subset of MHCII peptide complexes remains stably associated in the detergent SDS, but dissociate upon boiling into free MHCII $\alpha$ and $\beta$ chains (43). SDS-stable I-A $A^{g 7}$-peptide complexes are less prevalent than for most MHCII alleles; however, they can be detected (44). Thus, if the expression of DO in NOD DCs altered the I-A ${ }^{\mathrm{g}}$-bound peptide repertoire, there should be a detectable change in the level of SDS-stable I-Ag7 -peptide complexes. To test this idea, we performed immunoblotting of nonboiled and boiled lysates of NOD, NOD.DO, and C57BL/ 6 splenic DCs and B cells. A polyclonal rabbit serum against the cytoplasmic tail of I-A $\beta$ was used for the detection of $\mathrm{I}_{-} \mathrm{Ag}^{7}$-peptide complexes to ensure the detection of all SDS-stable complexes. C57BL/6 DCs were included as a positive control, since a large percentage of I- $A^{b}$-peptide complexes are known to remain stable in SDS (Figure 8B; band labeled " $\alpha \beta$ pep"). Both NOD DCs and B cells had detectable levels of SDS-stable I-Ag7-peptide complexes. This level, however, was clearly reduced in NOD.DO DCs (Figure 8B). Quantification of multiple experiments showed that the level of SDS-stable complexes in NOD.DO DCs was down approximately $60 \%$ relative to the level observed for NOD DCs (Figure 8C). B cells from NOD.DO mice also had a slight reduction $(-15 \%)$ in the level of SDS-stable dimers compared with NOD B cells (Figure 8C). Collectively, these studies show that DO expression in NOD DCs resulted in the presentation of an altered $\mathrm{I}-\mathrm{A}^{\mathrm{g}}{ }^{7}$-peptide repertoire by NOD.DO DCs. $B$ cells from NOD.DO mice also displayed an altered I-Ag $\mathrm{A}^{\mathrm{7}}$-peptide repertoire but to a much lesser extent than NOD.DO DCs.

\section{Figure 6}

NOD and NOD.DO mice have similar NP-specific antibody responses. $\lg \mathrm{M}(\mathbf{A})$ and $\lg \mathrm{G}(\mathbf{B})$ responses to NP were measured by ELISA on days $0,7,14$, and 54 after immunization of $\operatorname{NOD}(n=6)$ and NOD.DO $(n=6)$ mice with NP-CGG in alum. Each symbol represents an individual mouse, and horizontal bars represent the mean values for each group. $P$ values were computed using 2-tailed, unpaired Student's $t$ test and showed no significance differences between NOD and NOD.DO mice.

\section{Discussion}

Peptide loading of MHCII molecules is directly catalyzed by DM, which also edits the MHCII-bound peptide repertoire and functions as an MHCII-specific chaperone. While the essential function of DM in shaping the MHCII peptide repertoire is well established, the ramification of DO modulation of DM function in vivo remains enigmatic. Generally, it is accepted that DO reduces or modulates the complexity or level of the MHCII self peptide repertoire. This leads to the attractive hypothesis that DO dampens the Ag-processing ability of DCs and B cells to reduce the possibility of activating self-reactive CD4 T cells and thereby contributes to the maintenance of peripheral tolerance and protection from autoimmunity.

The studies presented here show that DO expression in DCs prevents autoimmune diabetes development in NOD mice. DO inhibits or modulates DM/H2-M-mediated peptide loading (12-14). Thus, the most plausible mechanism for protection from diabetes in NOD.DO mice is that DO blocks the presentation of isletderived peptides, thereby preventing the activation of pathogenic $\mathrm{T}$ cells. However, before drawing this conclusion, it was necessary to test other possible mechanisms for diabetes protection, such as altered central tolerance or enhanced Treg activity. Additionally, since DO modulates Ag presentation, it was possible that DO expression in NOD DCs created an overall immunosuppressive environment, which would prevent diabetes development.

Central tolerance is mediated by the deletion of the majority of autoreactive $\mathrm{T}$ cells in the thymus during development (45). In NOD mice, defects in central tolerance are believed to result in a significant number of self-reactive $T$ cells escaping to the periphery, which contributes to diabetes development (46). The expression of DO in DCs of NOD mice, therefore, may also alter negative selection, which would result in protection from diabetes. Our studies showed that BDC2.5 TCR Tg T cells, which recognize an unknown islet-derived self peptide in the context of the $\mathrm{I}^{\mathrm{g}} \mathrm{A}^{\mathrm{g}}$, were selected properly in BDC2.5/NOD.DO mice (Figure 4, A and B). In addition, similar numbers of BDC2.5-like $\mathrm{T}$ cells accumulated in the PLNs of NOD and NOD.DO animals (Figure 4A). These data, consequently, show that DO expression does not have an impact on the selection of at least one set of autoreactive $T$ cells. However, it is important to note that it is not clear whether the unknown islet recognized by BDC2.5 T cells represents an Ag that is critical for diabetes development. Indeed, our data support that it is not likely to be a diabetes-initiating Ag (Supplemental Figure 5). Although we can't completely rule out the possibility that the overall pool of T cells selected in NOD.DO mice is not identical to those selected in NOD mice, peripheral T cells from NOD.DO mice clearly have the capacity to transfer diabetes to immunocompromised NOD.scid recipients (Figure 3A). This finding shows that NOD.DO mice have a diabetogenic T cell repertoire. It is, however, not possible to definitively rule out that NOD.DO mice have an altered CD4 $\mathrm{T}$ cell repertoire and/or decreased numbers 
A
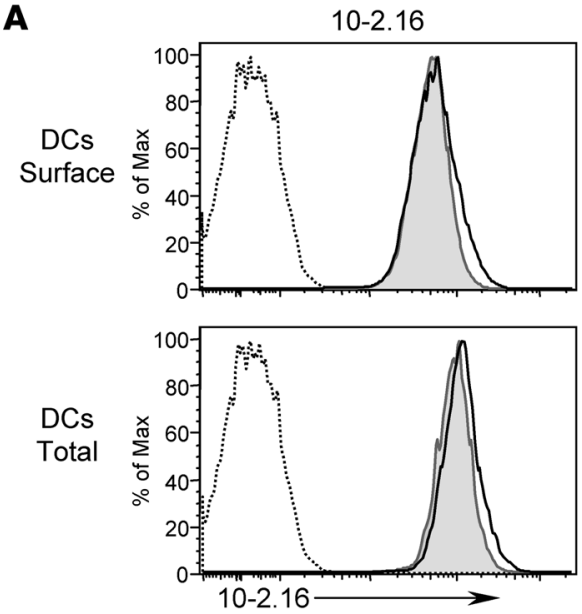

B

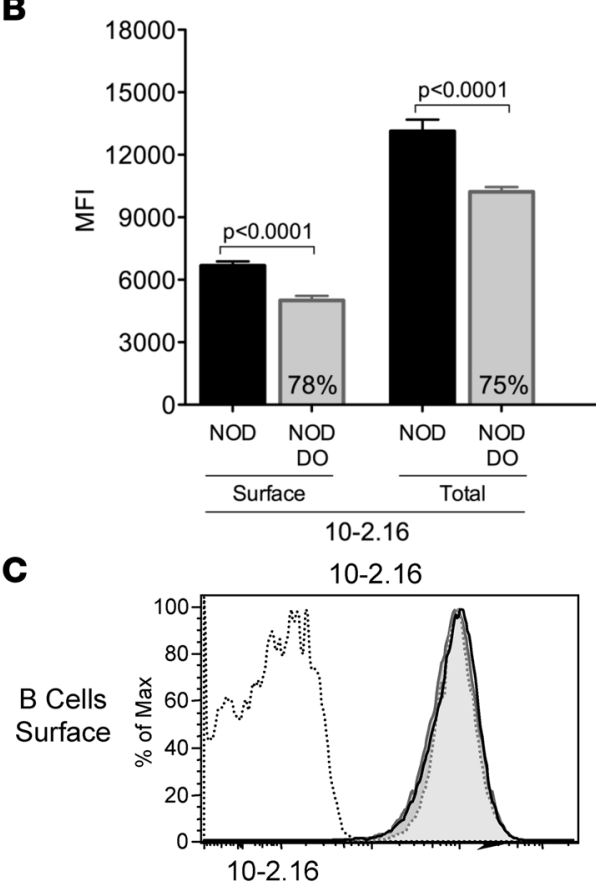

D

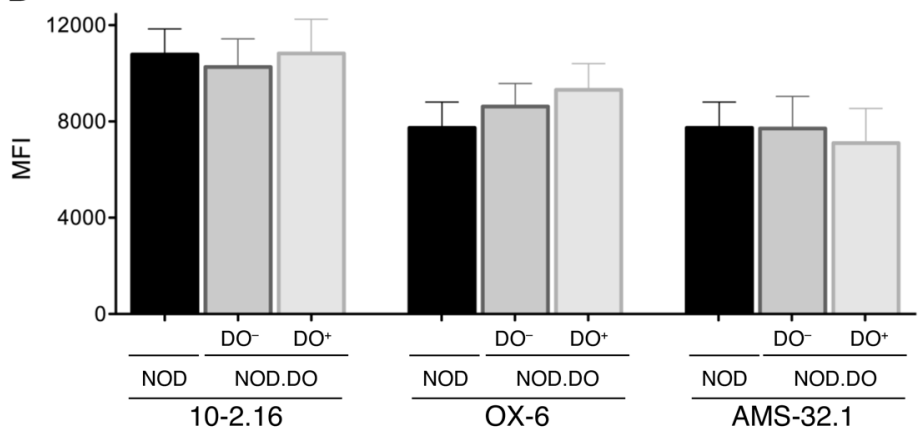

OX-6
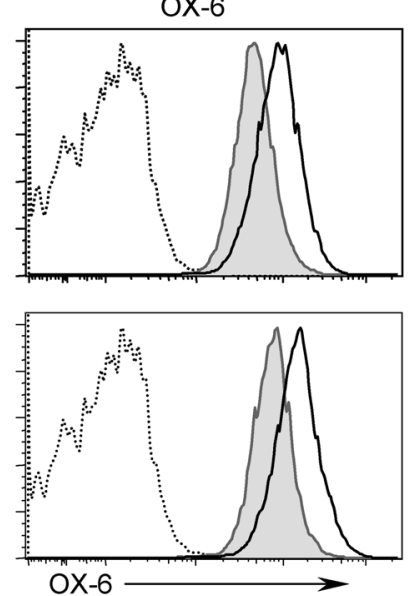

$p<0.0001$

\section{C}

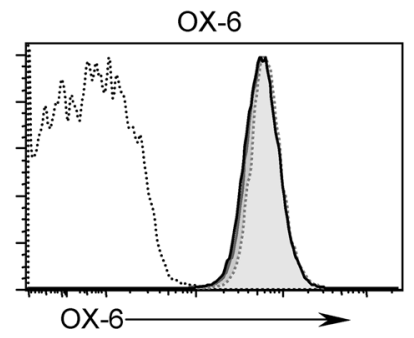

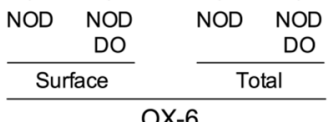

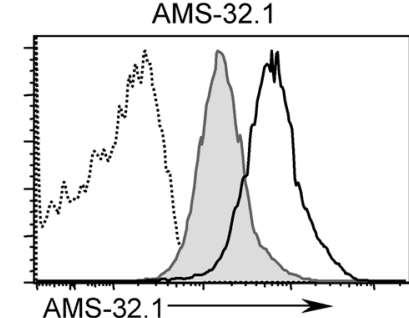

NOD

NOD.DO
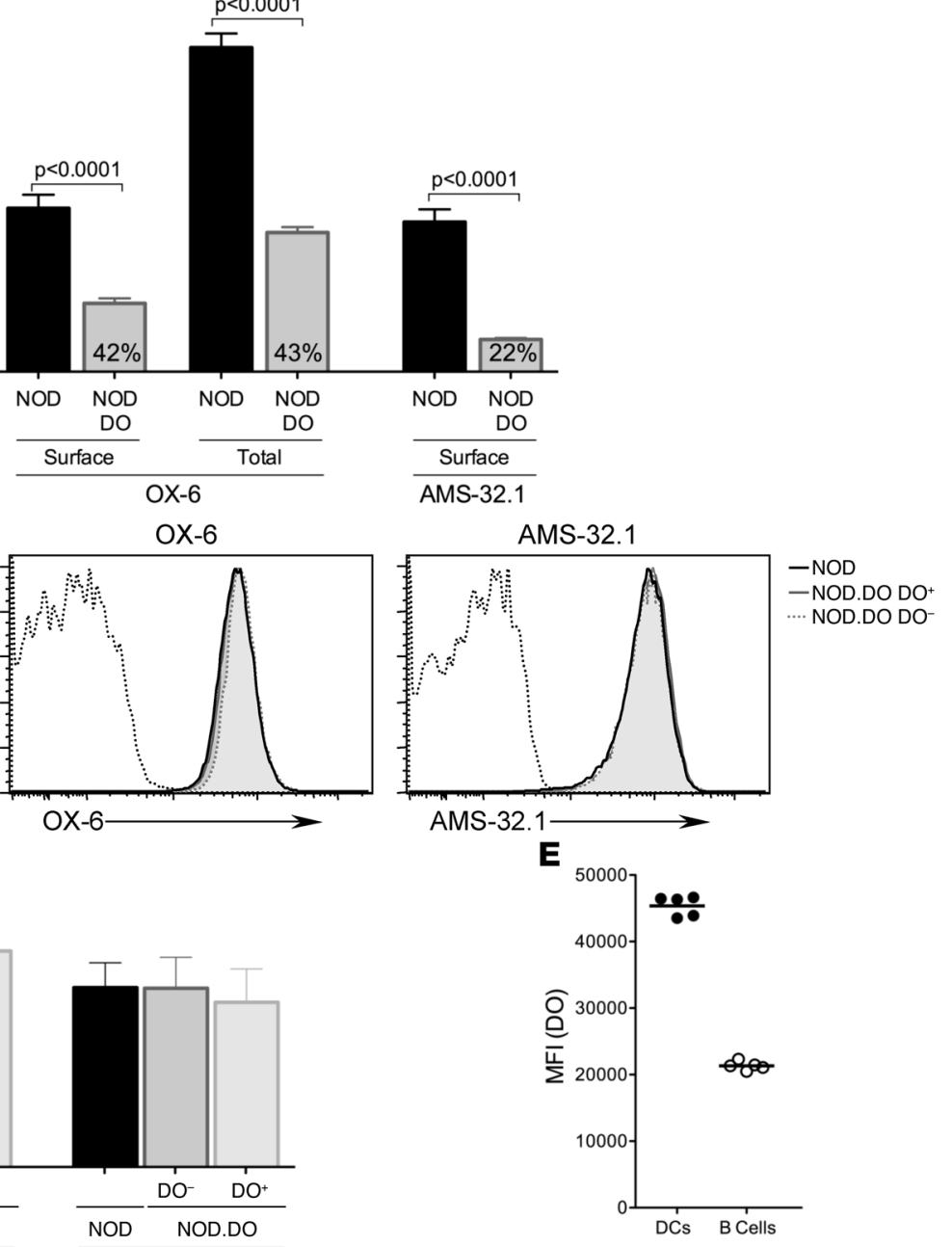


\section{Figure 7}

NOD.DO DCs display an altered MHCII peptide repertoire. (A) Splenocytes from NOD and NOD.DO mice were stained with a mAb specific for CD11c and the anti-MHCII mAbs 10-2.16, OX-6, and AMS-32.1. Top panel shows surface I-A ${ }^{97}$ levels for NOD and NOD.DO mice, and bottom panel shows surface and intracellular (total) $\mathrm{I}-\mathrm{A}^{97}$ levels obtained by permeabilizing cells after surface staining and restaining. (B) Quantification of 10-2.16, OX-6, and AMS-32.1 DC levels (MFI) obtained for multiple animals ( $n=6$ of each). Data represent mean \pm SEM. The numbers in the NOD.DO bar graphs represent the average MFI of NOD.DO DC I-Ag7 levels relative to that obtained for NOD. Data are representative of 4 (10-2.16), 2 (AMS-32.1), and more than 20 (OX-6) similar experiments. (C) Splenocytes from NOD and NOD.DO mice were stained with anti-CD19 and anti-MHCII mAbs 10-2.16, OX-6, and AMS-32.1, made permeable and stained with a mAb specific for DO. Histograms show $1-A^{97}$ levels in CD19+ $B$ cells from NOD mice and $\mathrm{DO}^{+}$and DO- CD19+ $\mathrm{B}$ cells from NOD.DO mice (determined by gating on $B$ cells staining positive or negative for DO). (D) Quantification of 10-2.16, OX-6, and AMS-32.1 surface B cell levels (MFI) obtained for multiple animals ( $n=4$ of each). Data represent mean \pm SEM. Data are representative of 2 similar experiments. (E) DO expression levels (MFI) in DO+ B cells and DCs from NOD.DO mice. Each symbol represents an individual mouse $(n=5)$, and horizontal bars represent the means. Data are representative of 3 similar experiments.

of pathogenic T cells and that these differences might contribute to the protection of NOD.DO mice from T1D.

Tregs play an important role in controlling the development of TID in NOD mice. Genetic depletion of Tregs accelerates diabetes development, whereas the transfer of Tregs into NOD recipients can delay diabetes onset (1). NOD Tregs show a defect in a standard in vitro suppression assay; however, a recent study shows that the main functional problem seems to lie in the ability of the NOD effector $\mathrm{T}$ cells to be regulated by Tregs (47). Mice deficient in the expression of the Ii or cathepsin L, 2 molecules involved in the MHCII Ag presentation pathway, were shown to be protected from diabetes $(48,49)$. The Ii is an essential MHCII chaperone that promotes MHCII surface expression, Ag presentation, and efficient CD4 $\mathrm{T}$ cell selection (11). Cathepsin $\mathrm{L}$ is a lysosomal protease that is involved in generating peptides that are presented by MHCII molecules as well as mediating Ii cleavage in the thymic epithelial cells (50). Protection from diabetes in Ii- and cathepsin L-deficient mice was mediated by an alteration in the balance of effector to Tregs caused by reduced conventional CD4 T cell numbers (48, 49). Although NOD.DO mice also have altered MHCII Ag presentation, we found no alteration in Treg or CD4 $\mathrm{T}$ cell percentages or absolute cell numbers (Supplemental Figure 2A and Supplemental Figure 4) and there also was no significant alteration of the ratio of absolute Treg/CD4 T cell numbers in the PLNs, spleen, or thymus of NOD and NOD.DO mice (data not shown). Furthermore, Foxp3 levels in Tregs from NOD.DO and NOD mice are similar (data not shown), supporting that NOD.DO and NOD Tregs function equivalently. Indeed, NOD.DO Tregs are functional, as shown by earlier diabetes onset in NOD.scid recipients that received NOD. DO T cells depleted of Tregs (Figure 3B). Collectively, our data support that protection from diabetes in NOD.DO mice is unlikely to be caused by alterations in Treg function.

Although diabetes in NOD mice is primarily driven by CD4 and CD8 T cells, B cells also play a crucial, but poorly understood role, perhaps by secreting antibody that facilitates disease initiation and/or by supporting the expansion of diabetogenic CD4 T cells by the presentation of islet-derived autoAgs $(1,51)$. Ag-specific B cells are efficient at presenting on MHCII nonabundant Ags such as islet-derived Ags internalized by the B cell receptor. Furthermore, it has been suggested that B cells present a different subset of MHCII peptide complexes than CD4 T cells and that this might contribute to disease amplification via epitope spreading (52). Transgenic DO expression in NOD.DO mice was not completely restricted to DCs. Approximately $20 \%$ of the total B cells also expressed the DO transgenes, with the majority of the DO-expressing $\mathrm{B}$ cells being MZ B cells. Our analysis of an additional NOD.DO Tg mouse line showed DO expression in only $10 \%$ of the B cells (Supplemental Figure 3). This line is also protected from diabetes, suggesting that expression of DO in B cells is not likely to be the main mediator of protection from diabetes. Furthermore, the transfer of pathogenic T cells into NOD.RAG1.DO mice, which lack DO-expressing $\mathrm{B}$ cells, results in the protection of these mice from diabetes. Thus, DO expression in DCs is sufficient to mediate protection.

Our studies support that DO expression most likely prevents diabetes in NOD mice by inhibiting the DC-mediated presentation of the islet-specific auto $\mathrm{Ag}(\mathrm{s})$ that are essential for disease initiation. DO could block diabetes by several different mechanisms. First, DO may prevent the presentation of a potential primary initiating Ag, such as insulin (53). By doing so, the disease would be blocked at the initial stage. Alternatively, if diabetes initiation is driven by multiple autoAgs, DO expression in DCs might inhibit the presentation of all the Ags or, more likely, only a subset of the self-Ags. The second situation is more plausible, since it has been shown that $\mathrm{DO} / \mathrm{H} 2-\mathrm{O}$ downmodulates the presentation of peptides derived from some, but not all Ags (19, 24, 54), an idea supported by our finding that BDC2.5 T cells proliferated well in NOD.DO mice. Importantly, these studies also showed that $\mathrm{DO} / \mathrm{H} 2-\mathrm{O}$ expression did not completely inhibit presentation, but reduced the level of specific MHCII peptide complexes $(19,24,54)$. Hence, DO expression in NOD.DO mice most likely decreases the presentation of islet-derived peptides, but does not completely inhibit presentation. The low level of MHCII-islet peptide complexes on the surface of DCs in NOD.DO mice may be insufficient to fully drive optimal $\mathrm{T}$ cell activation. This idea is supported by the finding that NOD.DO mice exhibited a block in diabetes development at the stage between benign periinsulitis and destructive insulitis. Identification of the autoAgs that are targets of DO downmodulation in NOD.DO mice would allow these questions to be evaluated as well as potentially identify diabetes-initiating auto $\mathrm{Ag}(\mathrm{s})$.

What is the in vivo function of DO/H2-O? Current untested ideas favor that DO/H2-O expression is important for maintaining central and peripheral tolerance $(15,17,23)$. In resting B cells, immature DCs, and thymic epithelium, DO/H2-O expression functions to dampen DM/H-2M activity, resulting in a potentially broad MHCII-bound peptide repertoire containing low levels of each individual self peptide that would promote peripheral $\mathrm{T}$ cell tolerance. Upon Ag-presenting cell activation, however, $\mathrm{DO} / \mathrm{H} 2-\mathrm{O}$ protein expression is rapidly downregulated, releasing the brakes and fully activating DM/H2-M to focus the MHCII peptide pool such that Ag-specific MHC peptide complexes are optimally presented for T cell activation. This model predicts, of course, that DO/H2-O expression would be necessary for tolerance induction and protection from autoimmune diseases. As such, H2-O-deficient mice would be expected to show signs of autoimmunity, but this has not occurred, at least on a C57BL/6 background (W. Yi and L.K. Denzin, unpublished results). The 
A

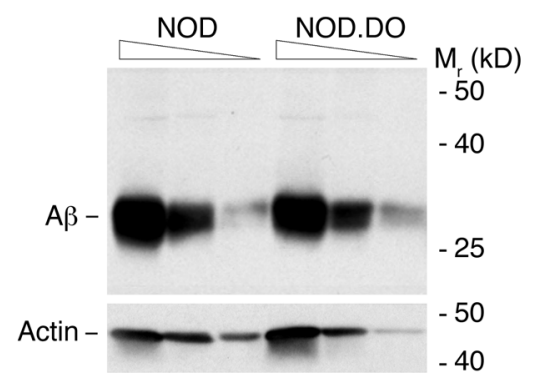

B

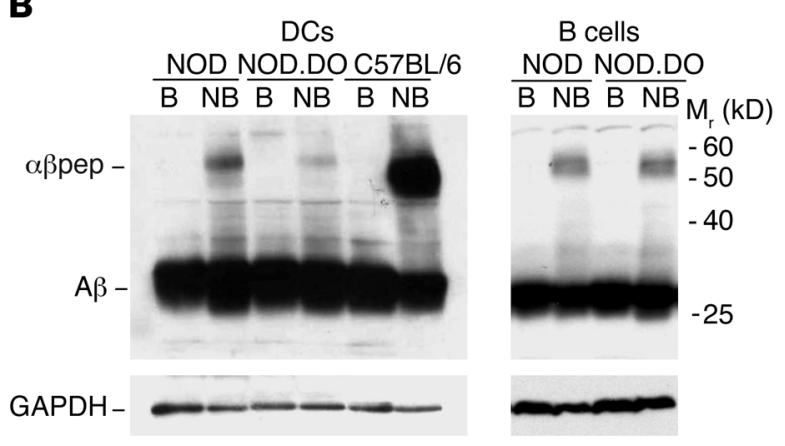

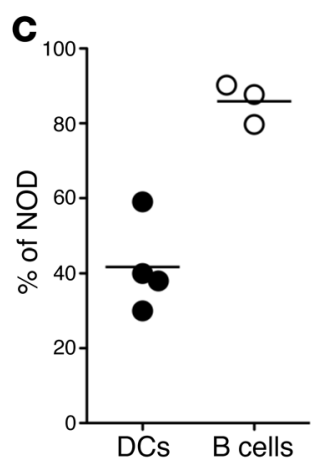

Figure 8

I-A ${ }^{97}$ molecules in NOD.DO DCs have reduced levels of SDS-stable dimers. (A) Titrated amounts of detergent lysates $\left(20,6.67\right.$, and $2.22 \times 10^{4}$ cell equivalents/lane) from purified NOD and NOD.DO splenic DCs were separated by SDS-PAGE, transferred to membranes, and probed with a rabbit sera specific for the cytoplasmic tail of I-A $\beta$. To demonstrate proportional loading, the blot was also probed with a mAb specific for actin. Data are representative of 3 independent experiments. (B) Detergent lysates from purified splenic DCs $\left(2.5 \times 10^{5}\right.$ cell equivalents/lane) or B cells $\left(5 \times 10^{5}\right.$ cell equivalents/lane) were incubated in sample buffer at room temperature (nonboiled; NB) or boiled (B) prior to separation by SDS-PAGE and transfer to membrane. Blots were probed with a polyclonal rabbit anti-sera to the cytoplasmic tail of I-A $\beta$. Probing of the blot with a mAb specific for GAPDH demonstrated equal loading. B6 DCs are included as a positive control for MHCIl peptide complex formation ( $\alpha \beta p e p)$. Data are representative of 4 (DCs) and 3 (B cells) independent experiments. (C) Quantification of the level of I-A ${ }^{97}$ SDS-stable dimers in NOD.DO DCs or B cells relative to NOD DCs or B cells, respectively. Each symbol represents an individual experiment and small horizontal bar indicates the mean.

results presented herein, however, clearly demonstrate that DO expression prevents autoimmune diabetes in NOD mice, strongly supporting a role for $\mathrm{DO} / \mathrm{H} 2-\mathrm{O}$ in the maintenance of tolerance. DO expression may function to further broaden the steady-state MHCII peptide repertoire, effectively reducing the level of diabetes-promoting peptides to a point that is no longer effective at inducing $\mathrm{T}$ cell activation. A recent study using green fluorescent protein as a surrogate islet-specific Ag nicely demonstrated a DCdriven amplification loop during the progression to T1D in NOD mice (5). These studies showed that initial $\mathrm{T}$ cell infiltration of pancreatic islets leads to intraislet DC maturation, DC migration to the PLN, and increased priming of more autoreactive $\mathrm{T}$ cells. It is possible that DO expression interrupts this amplification loop. Under normal circumstance, DO/H2-O expression is downregulated upon DC maturation $(15,17)$. However, our previous studies showed that in CD11c-DO Tg mice, DO levels do not significantly decrease following DC maturation (24). Thus, in NOD.DO mice, the initial $\mathrm{T}$ cell infiltrate may induce inflammation and subsequent DC maturation; however, DCs in NOD.DO mice would not downregulate DO expression, and although DCs may still migrate to the PLN, they would not arrive with optimum levels of MHCII peptide for T cell activation. Alternatively, DO expression might prevent the initial DC activation due to inadequate initial $\mathrm{T}$ cell priming.

Much attention has been placed on manipulating the $\mathrm{T}$ cell populations in NOD mice to treat and prevent disease (55). In contrast, little effort has been made toward altering Ag presentation by DCs in an effort to prevent and treat diabetes. Presumably, the generalized negative consequences of preventing Ag presentation in patients seemed insurmountable. Our finding that DO-mediated alteration of the MHCII pathway in DCs prevents autoimmune diabetes in NOD mice is a "proof of principle" that shows that subtle changes in MHCII presentation are sufficient for disease prevention and highlights the potential for manipulation of Ag presentation as a means to treat and prevent autoimmune disease.

\section{Methods}

Mice. NODLt/J and NOD.scid mice were purchased from the Jackson Laboratory. BDC2.5/NOD mice were received from the JDRF Center on Immunological Tolerance in T1D Resource and were bred and maintained in Memorial Sloan-Kettering Cancer Center's (MSKCC's) animal facilities. The generation of C57BL/6 CD11c-DO Tg mice has been described (24), and these were backcrossed more than 15 generations with NODLt/J. At the 15 th backcross generation, the linkage markers associated with Idd-recessive loci were confirmed to be NOD-derived by microsatellite analysis by Charles River Laboratories Genetic Testing Services. NOD.DO mice were maintained by crossing with NODLt/J mice, and for all experiments in which NOD.DO mice were compared with NOD mice, non-Tg littermate control mice were used. BDC2.5/ NOD.DO mice were generated by crossing BDC2.5/NOD with NOD.DO mice. All mice were housed under specific pathogen-free conditions in MSKCC animal facilities. All experimental procedures were approved by the Institutional Animal Care and Use Committee of MSKCC. Diabetes was monitored by the measurement of urine glucose levels with Diastix (Bayer). Mice were considered diabetic after 2 positive measurements over $250 \mathrm{mg} / \mathrm{dl}$, and onset of diabetes was recorded as the day of the first positive measurement. Female mice were used for all experiments except where noted.

Antibodies. The following fluorochrome or biotin-labeled mAbs were used for FACS analyses: anti-CD11c (HL3), anti-CD23 (FcERII; B3B4), anti-CD21/CD35 (7G6), anti-MHCII (AMS-32.1), anti-CD45R (B220; RA33-6B2), anti-rat MHCII (OX-6), anti-CD19 (1D3), anti-CD4 (RM4-5), anti-CD8 $\alpha$ (53-6.7), anti-CD25 (PC61), anti-TCR V $\beta 4$ (KT4), antiFoxp3 (FJK-16s), and anti-CD3 (17A2). These were from BD Biosciences - Pharmingen or eBioscience. Biotinylated mAbs were detected with APC-CY7-conjugated (Molecular Probes), APC- or PerCPCy5.5-conjugated (BD Biosciences - Pharmingen) streptavidin. Purified anti-CD25 (PC61) and anti-CD16/CD32 (Fcy block; 2.4G2) were purchased from MSKCC's Monoclonal Antibody Core Facility at MSKCC. Anti-DO mAb Mags.DO5 (21) and anti-MHCII mAb 10-2.16 were purified from bioreactor supernatants using standard protein G Sepharose (Amersham Pharmacia Biotech) affinity chromatography and used as purified mAbs 
or conjugated with Alexa Fluor 647 (Molecular Probes) according to the supplied protocol. The rabbit anti-I-A $\beta$ cytoplasmic tail Ab was a gift of Alexander Rudensky (MSKCC).

Flow cytometry. Freshly isolated cells from mechanically disrupted spleens, lymph nodes, or thymuses were depleted of erythrocytes by hypotonic lysis, washed with PBS containing 1\% fetal bovine serum and $10 \mathrm{mM}$ EDTA ( $\mathrm{pH}$ 7.4) (staining buffer), and blocked in staining buffer containing $1 \mu \mathrm{g} / \mathrm{ml} \mathrm{F}$ c $\gamma$ block and $10 \%$ normal mouse serum on ice for 15 minutes. Cells were then stained on ice for 20 to 30 minutes by the addition of specific antibodies. When biotinylated mAbs were used, a second staining with fluorochorome-labeled streptavidin for $15 \mathrm{~min}$ utes on ice was performed. Stained cells were analyzed using a BD 4-laser LSRII flow cytometer (BD Biosciences) and analyzed with FlowJo software. Dead cells were excluded from analysis by the addition of the cell vital dye DAPI. For intracellular staining, cells were incubated with $\mathrm{mAbs}$ specific for surface proteins and then fixed and permeabilized with Cytofix/Cytoperm (BD Biosciences - Pharmingen). After permeabilization, cells were stained for intracellular proteins. FACS staining of splenic DCs was performed following digestion of spleens in $400 \mathrm{U} / \mathrm{ml}$ collagenase D (Roche Applied Science) for 30 minutes at $37^{\circ} \mathrm{C}$.

Histology. Excised pancreata from NOD.DO and nTg littermate control NOD mice were fixed in $4 \%$ formaldehyde, embedded in paraffin, and sectioned prior to H\&E staining by MSKCC's Laboratory of Comparative Pathology. The total number of islets per section was counted, and the severity of lymphocyte invasion was scored for each islet. Noninvasive infiltration surrounding the islets was scored as periinsulitis. Infiltration of the pancreatic islet was scored as insulitis. The percentages of insulitis and periinsulitis were calculated by dividing the number of islets with periinsulitis or insulitis by the total number of islets examined. Three sections per mouse were scored.

Cell transfer studies. For adoptive transfers into NOD.scid recipients, $\mathrm{T}$ cells from 22-week-old NOD.DO and littermate non-Tg NOD mice were purified by negative selection with a Pan T cell Isolation Kit (Miltenyi Biotec). T cell purity was greater than $95 \%$. Purified T cells $\left(5 \times 10^{6} /\right.$ mouse $)$ were transferred into 8-week-old NOD.scid recipient mice by retroorbital injection. CD25-depleted T cells were purified by staining the lymphocytes with anti-CD25 biotin prior to isolation of the T cells using the Pan $\mathrm{T}$ cell Isolation Kit. Any remaining contaminating $\mathrm{CD} 25^{+}$cells were inactivated in vivo by the intraperitoneal injection of anti-CD25 (PC61; 400 $\mu \mathrm{g} /$ mouse) at the time of cell transfer. Recipient mice were maintained on Sulfatrim-supplemented water for the duration of the experiment. Urine glucose levels were monitored twice a week for 10 weeks.

To transfer diabetes from diabetic NOD mice to NOD.DO mice, total T cells were purified as described above from NOD within 2 weeks of testing positive for glucose in their urine. Purified T cells $\left(5 \times 10^{6}\right.$ cells/mouse $)$ were transferred into sublethally irradiated (7.75 Gy), 6- to 10 -week-old recipient NOD.DO and non-Tg littermate control NOD mice via retroorbital injection. Recipient animals were monitored for diabetes by measurement of urine glucose levels twice a week. Transfers into NOD.RAG1 and NOD.RAG1.DO mice were performed as above, with the exception that recipient mice were not irradiated prior to cell transfer.

In vivo proliferation of BDC2.5 T cells. BDC $2.5 \mathrm{~T}$ cells were purified by negative selection from the spleens and lymph nodes of BDC2.5 Tg NOD or NOD.DO mice using a CD4 T Cell Isolation Kit (Miltenyi Biotec) and labeled with CFSE as described (26). The CFSE-labeled T cells were resuspended at $2.5 \times 10^{7} / \mathrm{ml}$ in PBS, and 2 to $5 \times 10^{6}$ cells were injected into NOD or NOD.DO recipients via retroorbital injection. Three days after transfer, pancreatic and inguinal LNs were harvested and $\mathrm{CFSE}^{+} \mathrm{V} \mathrm{B} 4^{+} \mathrm{CD} 4^{+}$cells were analyzed by flow cytometry for dilution of the CFSE signal.
Tetramer staining. Biotinylated $\mathrm{A}^{\mathrm{g} 7}$ loaded with the BDC13 (AAVRPLWVRMEAA) or CLIP (PVSQMRMATPLLMRP) peptides were tetramerized by incubating with 4:1 ratio of $\mathrm{Ag}^{\mathrm{g}}$ peptide/PE streptavidin (Molecular Probes) for 1 hour at $4{ }^{\circ} \mathrm{C}(30)$. PLN cells pooled from 5 NOD or NOD. $\mathrm{DO}$ (5- to 7-week-old) mice were stained with the $\mathrm{A}^{\mathrm{g} 7}$ tetramers in RPMI supplemented with $10 \%$ fetal bovine serum for 30 minutes at room temperature with gentle rocking followed by an additional incubation for 20 minutes on ice with PerCP Cy5.5-CD4 and PE-Cy7-CD8, PE-Cy7-B220, and Pacific Blue-CD3. The stained lymphocytes were washed extensively, and magnetic enrichment of $\mathrm{I}-\mathrm{A}^{\mathrm{g}} \mathrm{t}^{7}$ tramer ${ }^{+}$cells was achieved by using anti-PE microbeads and separation on an MS column (Miltenyi Biotec). DAPI was added to the tetramer-enriched cells to allow for dead cell exclusion. The number of $\mathrm{A}^{\mathrm{g} 7}$ tetramer ${ }^{+}$cells $\left(\mathrm{CD}^{+} \mathrm{CD}^{+}{ }^{+}\right.$tetramer ${ }^{+} \mathrm{CD} 8^{-} \mathrm{B} 220^{-}$ DAPI- cells) was enumerated by flow cytometry.

Immunoblot analysis and quantitation. DCs and B cells were purified from male and female NOD and NOD.DO mice using Pan DC or CD19 Microbeads (Miltenyi Biotech) according to the supplied instructions. DCs and B cells were routinely more than $85 \%-95 \%$ pure. Purified DCs and B cells were extracted in $20 \mathrm{mM}$ Tris, $130 \mathrm{mM} \mathrm{NaCl}$, pH 8.0, containing 1\% Triton X-100 and Complete Protease Inhibitor Cocktail (Roche). Following the removal of nuclear material by centrifugation, lysates were mixed with $10 \times$ Laemmli sample buffer containing $20 \mathrm{mM}$ DTT and incubated at room temperature (nonboiled) or $95^{\circ} \mathrm{C}$ for 5 minutes (boiled) prior to separation by SDS-PAGE and transfer to PVDF membrane (Millipore). Membranes were incubated with rabbit Abs to the cytoplasmic tail of I-A $\beta$ followed by detection with HRP-conjugated goat anti-rabbit IgG (Jackson ImmunoResearch Laboratories). Blots were developed with SuperSignal West Pico chemiluminescent peroxidase substrate (Pierce Biotechnology) followed by exposure to film. To ensure equal loading of the DC and B cell lysates, membranes were reprobed with anti-actin (Sigma-Aldrich) or antiGAPDH (Millipore) Abs. Cell numbers used for each blot are indicated in the Figure 8 legend. For quantification of SDS-stable complexes, films were scanned and bands were quantitated using Quantity One software (Bio-Rad). The pixel density obtained for the I-A $\beta$ bands was normalized to that obtained for GAPDH, and the percentages of SDS-stable complexes were determined by dividing the relative number obtained for NOD.DO DCs or B cells by that obtained for NOD DCs or B cells.

Tcell-dependent antibody responses. 11-week-old male NOD.DO and non-Tg littermate control NOD mice were immunized intraperitoneally with $50 \mu \mathrm{g}$ of 4-hydroxy-3-nitrophenylacetyl (NP) coupled to chicken $\gamma$ globulin $\left(\mathrm{NP}_{36^{-}}\right.$ CGG; Biosearch Technologies) precipitated in alum. Serum from mice on days $0,7,14$, and 56 after immunization were quantified by ELISA to determine the relative levels of NP-specific IgM and IgG. ELISA plates were coated with $1 \mu \mathrm{g} / \mathrm{ml} \mathrm{NP}_{30}$-BSA (IgM) or $\mathrm{NP}_{3}$-BSA (IgG; Biosearch Technologies) overnight at $4^{\circ} \mathrm{C}$, washed, and blocked for 1 hour at room temperature with PBS containing $1 \%$ BSA and $0.1 \%$ Tween-20. Diluted sera (1:400; $\operatorname{IgM}$ and $1: 8,100 ; \operatorname{IgG}$ ) were added to the plates and incubated for 1 hour at room temperature. The plates were washed and incubated with HRP-conjugated anti-mouse IgM or IgG (Jackson ImmunoResearch Laboratories) for 1 hour at room temperature. Following washing, the HRP was detected by the addition of $3,3^{\prime}, 5,5^{\prime}$ tetramethyl benzidine substrate solution (BioFX) and quantitated by measuring the absorbance of each well at $450 \mathrm{~nm}$.

Statistics. All statistical analyses were performed using GraphPad Prism version 5.00 using an unpaired 2-tailed Student's $t$ test. Values of $P \leq 0.05$ were considered significant.

\section{Acknowledgments}

We thank Teresa Di Lorenzo, Mark Chong, Susan Wong, and members of the Denzin, Sant'Angelo, and Chaudhuri laboratories for helpful advice; Luis Ferran and H. Maggie O'Rourke for expert 
animal care; and Ming Li and Nicole Draghi for critical reading of the manuscript. This project was funded by a Juvenile Diabetes Research Foundation Innovative Research grant (5-2007-576) and NIH grant RO1-AI061484 to L.K. Denzin and by a JDRF Center on Immunological Tolerance in T1D at HMS grant (4-2007-1057; PI Diane Mathis) to K.W. Wucherpfennig.

1. Anderson MS, Bluestone JA. The NOD mouse: a model of immune dysregulation. Annu Rev Immunol. 2005;23:447-485.

2. Steinman RM, Hawiger D, Nussenzweig MC. Tolerogenic dendritic cells. Annu Rev Immunol. 2005;21:685-711.

3. Green EA, Flavell RA. The initiation of autoimmune diabetes. Curr Opin Immunol. 1999;11(6):663-669.

4. Turley S, Poirot L, Hattori M, Benoist C, Mathis D. Physiological beta cell death triggers priming of self-reactive $T$ cells by dendritic cells in a type- 1 diabetes model. J Exp Med. 2003;198(10):1527-1537.

5. Melli K, et al. Amplification of autoimmune response through induction of dendritic cell maturation in inflamed tissues. $J$ Immunol. 2009;182(5):2590-2600.

6. Tisch R, McDevitt H. Insulin-dependent diabetes mellitus. Cell. 1996;85(3):291-297.

7. Kanagawa O, Martin SM, Vaupel BA, Carrasco-Marin E, Unanue ER. Autoreactivity of T cells from nonobese diabetic mice: an I-Ag7dependent reaction. Proc Natl Acad Sci U S A. 1998;95(4):1721-1724.

8. Stratmann T, et al. The I-Ag7 MHC class II molecule linked to murine diabetes is a promiscuous peptide binder. J Immunol. 2000;165(6):3214-3225.

9. Schmidt D, Verdaguer J, Averill N, Santamaria P. A mechanism for the major histocompatibility complex-linked resistance to autoimmunity. J Exp Med. 1997;186(7):1059-1075.

10. Todd JA, Bell JI, McDevitt HO. HLA-DQ beta gene contributes to susceptibility and resistance to insulin-dependent diabetes mellitus. Nature. 1987;329(6140):599-604.

11. Hudson AW, Ploegh HL. The cell biology of antigen presentation. Exp Cell Res. 2002;272(1):1-7.

12. Denzin LK, Sant'Angelo DB, Hammond C, Surman MJ, Cresswell P. Negative regulation by HLA-DO of $\mathrm{MHC}$ class II-restricted antigen processing. Science. 1997;278(5335):106-109.

13. Liljedahl $\mathrm{M}$, et al. Altered antigen presentation in mice lacking H2-O. Immunity. 1998;8(2):233-243.

14. van Ham SM, et al. HLA-DO is a negative modulator of HLA-DM-mediated MHC class II peptide loading. Curr Biol. 1997;7(12):950-957.

15. Chen X, Reed-Loisel LM, Karlsson L,Jensen PE. H2-O expression in primary dendritic cells. J Immunol. 2006;176(6):3548-3556.

16. Fallas JL, Yi W, Draghi NA, O'Rourke HM, Denzin LK. Expression patterns of $\mathrm{H} 2-\mathrm{O}$ in mouse B cells and dendritic cells correlate with cell function. J Immunol 2007;178(3):1488-1497.

17. Hornell TM, et al. Human dendritic cell expression of HLA-DO is subset specific and regulated by maturation. J Immunol. 2006;176(6):3536-3547.

18. Liljedahl $\mathrm{M}$, et al. HLA-DO is a lysosomal resident which requires association with HLA-DM for efficient intracellular transport. $E M B O \mathrm{~J}$. 1996;15(18):4817-4824.

19. Denzin LK, Fallas JL, Prendes M, Yi W. Right place, right time, right peptide: DO keeps DM focused. Immunol Rev. 2005;207:279-292.

20. Chen X, et al. Regulated expression of human histocompatibility leukocyte antigen (HLA)-DO during antigen-dependent and antigen-independent phases of B cell development. J Exp Med.

Received for publication June 17, 2009, and accepted in revised form January 6, 2010.

Address correspondence to: Lisa K. Denzin, Sloan-Kettering Institute, 1275 York Avenue, Box 509, New York, NY 10065. Phone: 646.888.2333; Fax: 646.422.0470; E-mail: denzinl@mskcc.org.

\section{2;195(8):1053-1062}

21. Glazier KS, Hake SB, Tobin HM, Chadburn A, Schattner EJ, Denzin LK. Germinal center B cells regulate their capability to present antigen by modulation of HLA-DO.J Exp Med. 2002;195(8):1063-1069.

22. Roucard C, et al. In vivo and in vitro modulation of HLA-DM and HLA-DO is induced by B lymphocyte activation. J Immunol. 2001;167(12):6849-6858.

23. Jensen PE. Antigen processing: HLA-DO--a hitchhiking inhibitor of HLA-DM. Curr Biol. 1998;8(4):R128-131.

24. Fallas JL, Tobin HM, Lou O, Guo D, Sant'Angelo DB, Denzin LK. Ectopic expression of HLA-DO in mouse dendritic cells diminishes MHC class II antigen presentation. J Immunol. 2004;173(3):1549-1560.

25. Postigo AA, Corbi AL, Sanchez-Madrid F, de Landazuri MO. Regulated expression and function of CD11c/CD18 integrin on human B lymphocytes. Relation between attachment to fibrinogen and triggering of proliferation through CD11c/CD18. J Exp Med. 1991;174(6):1313-1322.

26. Hoglund P, Mintern J, Waltzinger C, Heath W, Benoist C, Mathis D. Initiation of autoimmune diabetes by developmentally regulated presentation of islet cell antigens in the pancreatic lymph nodes. J Exp Med. 1999;189(2):331-339.

27. Ohnmacht $\mathrm{C}$, et al. Constitutive ablation of dendritic cells breaks self-tolerance of CD4 T cells and results in spontaneous fatal autoimmunity. $J$ Exp Med. 2009;206(3):549-559.

28. Pugliese A, Diez J. Lymphoid organs contain diverse cells expressing self-molecules. Nat Immunol. 2002;3:335-336; author reply 336 .

29. Christianson SW, Shultz LD, Leiter EH. Adoptive transfer of diabetes into immunodeficient NOD-scid/scid mice. Relative contributions of CD4+ and CD8+ T-cells from diabetic versus prediabetic NOD.NON-Thy-1a donors. Diabetes. 1993;42(1):44-55.

30. Jang MH, Seth NP, Wucherpfennig KW. Ex vivo analysis of thymic CD4 T cells in nonobese diabetic mice with tetramers generated from I-A(g7)/class II-associated invariant chain peptide precursors. J Immunol. 2003;171(8):4175-4186.

31. Haskins K, Portas M, Bradley B, Wegmann D, Lafferty K. T-lymphocyte clone specific for pancreatic islet antigen. Diabetes. 1988;37(10):1444-1448.

32. Katz JD, Wang B, Haskins K, Benoist C, Mathis $D$. Following a diabetogenic $T$ cell from genesis through pathogenesis. Cell. 1993;74(6):1089-1100.

33. Bikoff EK, Kenty G, Van Kaer L. Distinct peptide loading pathways for MHC class II molecules associated with alternative Ii chain isoforms. J Immunol. 1998;160(7):3101-3110.

34. Bikoff EK, Wutz G, Kenty GA, Koonce CH, Robertson EJ. Relaxed DM requirements during class II peptide loading and CD4+ T cell maturation in BALB/c mice. J Immunol. 2001;166(8):5087-5098.

35. Fung-Leung WP, et al. Antigen presentation and $\mathrm{T}$ cell development in H2-M-deficient mice. Science. 1996;271(5253):1278-1281.

36. Kenty G, Bikoff EK. BALB/c invariant chain mutant mice display relatively efficient maturation of $\mathrm{CD} 4+\mathrm{T}$ cells in the periphery and secondary proliferative responses elicited upon peptide challenge. J Immunol. 1999;163(1):232-241.
37. Kenty G, Martin WD, Van Kaer L, Bikoff EK. MHC class II expression in double mutant mice lacking invariant chain and DM functions. J Immunol. 1998;160(2):606-614.

38. Koonce CH, Wutz G, Robertson EJ, Vogt AB, Kropshofer H, Bikoff EK. DM loss in k haplotype mice reveals isotype-specific chaperone requirements. J Immunol. 2003;170(7):3751-3761.

39. Miyazaki T, et al. Mice lacking H2-M complexes, enigmatic elements of the MHC class II peptideloading pathway. Cell. 1996;84(4):531-541.

40. Oi VT, Jones PP, Goding JW, Herzenberg LA. Properties of monoclonal antibodies to mouse Ig allotypes, H-2, and Ia antigens. Curr Top Microbiol Immunol. 1978;81:115-120.

41. Fukumoto T, McMaster WR, Williams AF. Mouse monoclonal antibodies against rat major histocompatibility antigens. Two Ia antigens and expression of Ia and class I antigens in rat thymus. EurJ Immunol. 1982;12(3):237-243.

42. Wall KA, Lorber MI, Loken MR, McClatchey S, Fitch FW. Inhibition of proliferation of MIs- and Ia-reactive cloned $\mathrm{T}$ cells by a monoclonal antibody against a determinant shared by I-A and I-E. J Immunol. 1983;131(3):1056-1064.

43. Germain RN, Hendrix LR. MHC class II structure, occupancy and surface expression determined by post-endoplasmic reticulum antigen binding. Nature. 1991;353(6340):134-139.

44. Carrasco-Marin E, Shimizu J, Kanagawa O, Unanue ER. The class II MHC I-Ag7 molecules from nonobese diabetic mice are poor peptide binders. J Immunol. 1996;156(2):450-458.

45. Gallegos AM, Bevan MJ. Central tolerance: good but imperfect. Immunol Rev. 2006;209:290-296.

46. Mathis D, Benoist C. Back to central tolerance. Immunity. 2004;20(5):509-516.

47. D'Alise AM, et al. The defect in T-cell regulation in NOD mice is an effect on the T-cell effectors. Proc Natl Acad Sci U S A. 2008;105(50):19857-19862.

48. Mellanby RJ, Koonce CH, Monti A, Phillips JM, Cooke A, Bikoff EK. Loss of invariant chain protects nonobese diabetic mice against type 1 diabetes. J Immunol. 2006;177(11):7588-7598.

49. Maehr R, et al. Cathepsin L is essential for onset of autoimmune diabetes in NOD mice. J Clin Invest. 2005;115(10):2934-2943.

50. Honey K, Rudensky AY. Lysosomal cysteine proteases regulate antigen presentation. Nat Rev Immunol. 2003;3(6):472-482.

51. Silveira PA, Grey ST. B cells in the spotlight: innocent bystanders or major players in the pathogenesis of type 1 diabetes. Trends Endocrinol Metab. 2006;17(4):128-135.

52. Dai YD, Carayanniotis G, Sercarz E. Antigen processing by autoreactive B cells promotes determinant spreading. Cell Mol Immunol. 2005;2(3):169-175.

53. Zhang L, Nakayama M, Eisenbarth GS. Insulin as an autoantigen in NOD/human diabetes. Curr Opin Immunol. 2008;20(1):111-118.

54. Alfonso C, et al. Analysis of $\mathrm{H} 2-\mathrm{O}$ influence on antigen presentation by B cells. J Immunol. 2003;171(5):2331-2337.

55 . Shoda LK, et al. A comprehensive review of interventions in the NOD mouse and implications for translation. Immunity. 2005;23(2):115-126. 